\title{
Generalizable density functional theory based photoemission model for the accelerated development of photocathodes and other photoemissive devices
}

\author{
Evan R. Antoniuk, ${ }^{1}$ Yumeng Yue, ${ }^{2}$ Yao Zhou, ${ }^{3}$ Peter Schindler $\odot,{ }^{2}$ W. Andreas Schroeder, ${ }^{4}$ Bruce Dunham, ${ }^{5}$ Piero Pianetta, \\ Theodore Vecchione, ${ }^{5}$ and Evan J. Reed ${ }^{2}$ \\ ${ }^{1}$ Department of Chemistry, Stanford University, Stanford, California 94305, USA \\ ${ }^{2}$ Department of Materials Science and Engineering, Stanford University, Stanford, California 94305, USA \\ ${ }^{3}$ Google, Mountain View, California 94043, USA \\ ${ }^{4}$ Department of Physics, University of Illinois at Chicago, Chicago, Illinois 60607, USA \\ ${ }^{5}$ SLAC, Menlo Park, California 94025, USA
}

(Received 12 February 2020; revised manuscript received 7 May 2020; accepted 19 May 2020; published 29 June 2020)

\begin{abstract}
In this work, we have developed an ab initio photoemission model that accurately describes the photoemission process for the most diverse range of photocathode materials to date. Compared to previous photoemission models, this is accomplished by considerably reducing the number of approximations and assumptions used in representing the photoemission process and the photoemitting material itself. Notably, our model directly includes the full electronic structure of the material, photoexcitation probabilities for all direct optical transitions, and an improved surface-vacuum barrier transmission probability. To test the performance of our model, we perform validations with experimental measurements for all photocathode materials studied in this work. Whereas previous models have often qualitatively disagreed with the measured photoemission properties of some materials, our model is found to provide quantitative agreement with experimental measurements for all tested materials. As an example, our method predicts the root-mean-square transverse momentum of electrons emitted from PbTe up to an excess energy of $1.0 \mathrm{eV}$ with a mean absolute error that is $\sim 5 \times$ less than from previously derived expressions. Perhaps more importantly, our model is able to match experimentally observed decreases in intrinsic emittance with increasing photon energy - a feat that current analytical models are unable to achieve. We expect that the broad applicability of our model will greatly accelerate the rate of discovery, characterization, and scientific understanding of photocathodes and other photonic devices.
\end{abstract}

DOI: 10.1103/PhysRevB.101.235447

\section{INTRODUCTION}

Accurate modelling of optical phenomena is vital for understanding and discovering innovative photonics materials for photodetectors and optical communication applications [1-3]. This is also especially true regarding the development of photocathode materials used in x-ray free-electron laser (FEL) light sources.

X-ray FELs are particle accelerators that use a beam of electrons to generate $\mathrm{X}$-rays. These $\mathrm{X}$-ray FEL light sources have recently enabled exciting discoveries. The hard x-ray FEL at SLAC National Accelerator Laboratory has enabled researchers to monitor bond formation in the active site of proteins [4], optically tune the interlayer interactions in two-dimensional materials [5], and probe the formation of diamonds from laser-compressed hydrocarbons [6]. Through

Published by the American Physical Society under the terms of the Creative Commons Attribution 4.0 International license. Further distribution of this work must maintain attribution to the author(s) and the published article's title, journal citation, and DOI. the further development of bright $\mathrm{x}$-ray FEL light sources, previously impossible experiments may be realized [7].

The electron beams used in x-ray FELs are typically generated by laser-driven photocathodes and the photocathodes are typically metals like copper, or commonly recognized photomultiplier tube materials such as $\mathrm{Cs}_{2} \mathrm{Te}, \mathrm{GaAs}$, or bialkali antimonides. The brightness of the electron beam is defined to be the number of electrons per unit time passing through a unit area. When electron beam brightness is normalized by the beam energy it is an invariant quantity, making it useful for evaluating the performance of electron-optical systems.

The normalized brightness of the electron beam is proportional to the inverse square of the variance in the momentum of electrons emitted in the transverse direction (parallel to the photocathode surface). When normalized by the beam energy, this quantity is called normalized transverse emittance, $\varepsilon_{n, x}$,

$$
\varepsilon_{n, x}:=\frac{1}{m c} \sqrt{\left\langle x^{2}\right\rangle\left\langle p_{x}^{2}\right\rangle-\left\langle x p_{x}\right\rangle^{2}},
$$

where $x$ and $p_{x}$ are the transverse position and transverse momentum of the emitted electrons, respectively. When studying photoemission, the position and momentum of the photoemitted electrons are uncorrelated when the beam is created in uniform extraction fields from a photocathode of uniform 
composition and surface geometry. Under these conditions, Eq. (1) simplifies to

$$
\varepsilon_{n, x}=\frac{1}{m c} \sqrt{\left\langle x^{2}\right\rangle\left\langle p_{x}^{2}\right\rangle} .
$$

According to Eq. (2), the normalized transverse emittance can be reduced by decreasing the size of the electron beam, $\sqrt{\left\langle x^{2}\right\rangle}$, resulting in an artificially higher normalized brightness. A more robust metric of photocathode performance is therefore the normalized transverse emittance divided by the size of the electron beam size, referred to as intrinsic emittance, $\varepsilon_{\text {int }}$ :

$$
\varepsilon_{\text {int }}:=\frac{\varepsilon_{n, x}}{\sqrt{\left\langle x^{2}\right\rangle}}=\frac{1}{m c} \sqrt{\left\langle p_{x}^{2}\right\rangle}=\frac{\hbar}{m c} \sqrt{\left\langle k_{x}^{2}\right\rangle}
$$

and depends only on the photocathode material properties [8].

In order to understand the role photocathodes play in FEL performance, a relationship can be derived that gives the power of $\mathrm{x}$ rays radiated from an $\mathrm{x}$-ray FEL as a function of the exponential of the inverse cube root of the normalized transverse emittance [9]. This illustrates that photocathode selection can greatly affect FEL performance. However, due to the complex nature of the photoemission process, the development of accurate models is a challenging task. Currently, photoemission models cannot be applied to universally predict the emission properties of photocathode materials [10,11], which limits our ability to discover new and better photocathodes.

The most common current basis for understanding photoemission is derived from the Spicer three-step model, consisting of (1) photoexcitation of electrons, (2) transport of these photoexcited electrons to the surface-vacuum interface, and (3) the transmission of these electrons into the vacuum [12]. By assigning a probability to each of these steps, the variance in the $x$ component (parallel to the photocathode surface) of the momentum of emitted electrons has previously been expressed as [13]

$$
\varepsilon_{\mathrm{int}}=\frac{\int_{E_{\mathrm{vacuum}}+\hbar \omega}^{\infty}[1-f(E+\hbar \omega)] f(E) d E \int_{\cos \left[\theta_{\max }(E)\right]}^{1} d(\cos \theta) \int_{0}^{2 \pi} p_{x}^{2} d \varphi \int_{0}^{\infty} G(s, E, \theta, \omega) d s}{m c^{2} \int_{E_{\mathrm{vacuum}}+\hbar \omega}^{\infty}[1-f(E+\hbar \omega)] f(E) d E \int_{\cos \left[\theta_{\max }(E)\right]}^{1} d(\cos \theta) \int_{0}^{2 \pi} d \varphi \int_{0}^{\infty} G(s, E, \theta, \omega) d s}
$$

This expression represents the probability of photoexcitation between two states as $[1-f(E+\hbar \omega)] f(E)$, where $f(E)$ is the energy density of occupied states at an energy $E$. This representation of the photoexcitation probability assumes that the photoexcitation probability only depends on the occupation of the initial and final states. The effect of electron transport is captured through the electron scattering term $G(s, E, \theta, \omega)$, which is defined as the fraction of electrons which survive scattering at a depth $s$ below the incident surface. The transmission of electrons into the vacuum is then expressed as $\int_{\cos \left[\theta_{\max }(E)\right]}^{1} d(\cos \theta)$, where $\theta_{\max }(E)$ is defined as the maximum escape angle for an electron with energy $E$.

By neglecting the angular dependence on the scattering probability $\theta$ and the energy dependence of the scattering mean free path, the scattering probability in the numerator and denominator of Eq. (4) cancel. By assuming the electronic temperature is $0 \mathrm{~K}$, and using the Heaviside step function to represent the density of occupied states, $f(E)$, Eq. (4) can be analytically integrated to give

$$
\varepsilon_{\text {int }}=\sqrt{\frac{\hbar \omega-\phi}{3 m c^{2}}},
$$

where $\phi$ is the effective work function of the photocathode, which includes the Schottky potential barrier reduction at the surface-vacuum interface. Equation (4) can also be integrated using the Fermi-Dirac distribution in place of the Heaviside step function to give

$$
\varepsilon_{\text {int }}=\sqrt{\frac{k T}{m c^{2}}} \sqrt{\frac{\mathrm{Li}_{3}\left(-e^{\frac{e}{k T}(\hbar \omega-\phi)}\right)}{\operatorname{Li}_{2}\left(-e^{\frac{e}{k T}(\hbar \omega-\phi)}\right)}},
$$

where $\operatorname{Li}_{n}(z)$ is the polylogarithm function of order $n$ [14]. When taking the limit of the electronic temperature $T$, going to $0 \mathrm{~K}$, Eq. (6) reduces to Eq. (5).
The model given in Eq. (5) has had limited success predicting the intrinsic emittance of the semiconducting alkalibased photocathodes $\mathrm{K}_{2} \mathrm{CsSb}$ [15] and $\mathrm{Cs}_{3} \mathrm{Sb}$ [11], but performed well for predicting the emittance of simple metallic photocathodes such as $\mathrm{Cu}$ and Mo [16,17]. However, the mean transverse energy of electrons emitted from $\mathrm{PbTe}(111)$ predicted with Eq. (5) was found to be $20 \times$ smaller than experimental measurements and failed to capture the observed decrease in emittance with increasing photon energy. This reflects the need for a new broadly applicable model for accurately predicting photoemission $[10,18]$.

In this work, we develop a density functional theory (DFT) based method to model the photoemission process. Most notably, this method expands on the capabilities of the model given in Eq. (5) by utilizing DFT-calculated density of states, rather than assuming constant density of states. Additionally, we incorporate the photoexcitation probabilities for all possible optical excitations and a more accurate electron transmission probability across the photocathode-vacuum interface. Increasingly more accurate physical representations may allow our model to be generalizable to a wider range of materials. Coupled with the relatively low cost of performing DFT calculations, the general nature of our model may enable the possibility of rapidly screening through thousands of novel photocathode materials.

\section{METHOD DEVELOPMENT}

\section{A. Background}

Evaluation of intrinsic emittance requires determining the rms transverse momentum of the distribution of emitted electrons [Eq. (3)]. In the present work, this is accomplished by utilizing Kohn-Sham density functional theory $[19,20]$ (KSDFT) to calculate the ground-state Kohn-Sham states, $\psi_{\vec{k}, n}(\vec{r})$, 


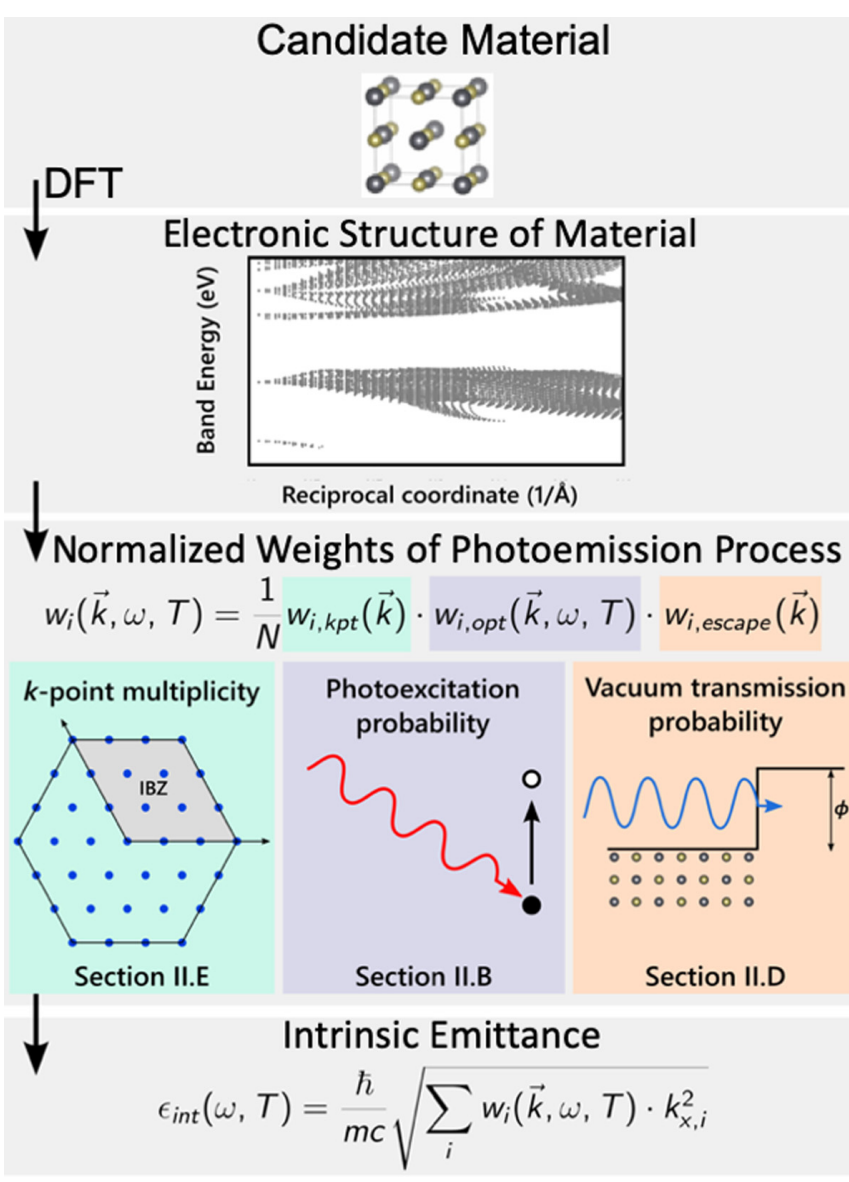

FIG. 1. Workflow for calculating the intrinsic emittance for a given material. We first obtain the relaxed crystal structure of the bulk material. We then calculate the electronic structure of the material with KS-DFT. Finally, we determine the total escape probability $\left(w_{i}\right)$ associated with all Kohn-Sham states and calculate the intrinsic emittance, $\varepsilon_{\text {int }}$.

where $\vec{k}$ is any reciprocal space vector in the first Brillouin zone and $n$ is the band index. The general workflow for calculating the intrinsic emittance of a photocathode material is summarized in Fig. 1.

To greatly aid in the computational efficiency of this calculation, we filter the Kohn-Sham states, $\psi_{\vec{k}, n}(\vec{r})$, that cannot contribute to the distribution of electrons emitted in the transverse direction. First, we filter out all $\vec{k}$ vectors orthogonal to the surface-normal direction, which we here define to be along the $z$ axis. This requirement is equivalent to requiring all photoexcited electrons to have $\left|k_{z}\right|>0$ as is necessary for transmission across the photocathode-vacuum interface. Next, for a photon of energy, $\hbar \omega \geqslant \phi$, a conduction-band state with energy $E_{c}$, and a valence-band state with energy $E_{v}$, we exclude all pairs of states which satisfy $E_{c}-E_{v}>$ $\hbar \omega$ and all conduction-band states that satisfy $E_{c}<E_{\mathrm{vacuum}}$, where $E_{\text {vacuum }}=E_{\mathrm{Fermi}}+\phi$. This filtering process is depicted graphically in Fig. 2 and is used for both metals and semiconductors. The first condition ensures that the incident photon is energetic enough to induce a photoexcitation between the two states, while the second condition ensures that the photoexcited electrons have enough energy to escape to the pho-

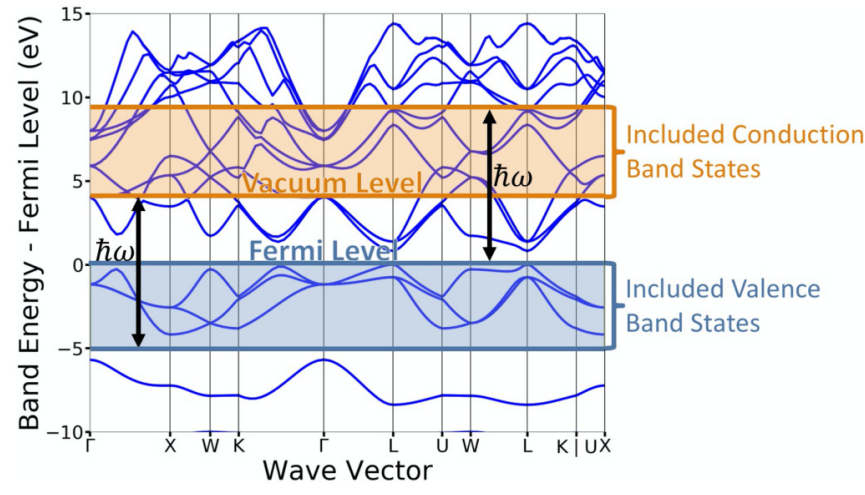

FIG. 2. Calculated PbTe electronic band structure. For an incident photon of energy, $\hbar \omega$, only electrons excited into the conduction-band states with energy above the vacuum level, $E_{\text {vacuum }}=E_{\mathrm{Fermi}}+\phi$, have sufficient energy to escape the photocathode surface-vacuum interface. The maximum energy a conductionband state can have to undergo photoexcitation is $\hbar \omega$, relative to the Fermi level. As such, the relevant conduction-band states have energy $E_{c}$ between $E_{\text {vacuum }} \leqslant E_{c} \leqslant \hbar \omega$. The minimum energy that a valence-band state must have to undergo photoexcitation followed by emission to vacuum is given by $E_{\text {vacuum }}-\hbar \omega$. The above plot of the PbTe electronic band structure shows all Kohn-Sham states that may contribute to photoemission with a photon energy of $9 \mathrm{eV}$ and a vacuum level of $4 \mathrm{eV}$, relative to the Fermi level.

tocathode surface-vacuum interface. The details of these two processes are discussed in Secs. II B and II D, respectively.

Following this filtering process, we obtain $i$ pairs of conduction-band states and valence-band states that satisfy $E_{c}-E_{v} \leqslant \hbar \omega$ and $E_{c}>E_{\mathrm{vacuum}}$ and exhibit the same $\vec{k}$. The contribution of each pair of states to the intrinsic emittance is then calculated according to

$$
\varepsilon_{\text {int }}(\omega, T)=\frac{\hbar}{m c} \sqrt{\sum_{i} w_{i}(\vec{k}, \omega, T) k_{x, i}^{2}},
$$

where $w_{i}$ is the normalized weight associated with the $i$ th possible optical transition from a valence-band state to a conduction-band state. In turn, we calculate $w_{i}$ as

$$
\begin{aligned}
w_{i}(\vec{k}, \omega, T)= & \frac{1}{N} w_{i, \mathrm{kpt}}(\vec{k}) \\
& \times w_{i, \mathrm{opt}}(\vec{k}, \omega, T) w_{i, \text { escape }}(\vec{k}),
\end{aligned}
$$

where $w_{i, \mathrm{kpt}}(\vec{k})$ is the number of $k$ points in the Brillouin zone that contribute equally to the total photoemission, $w_{i, \text { opt }}(\vec{k}, \omega, T)$ is the photoexcitation probability, $w_{i, \text { escape }}(\vec{k})$ is the vacuum transmission probability, and $N$ is a normalization factor given by $N=\sum_{i} w_{i, \mathrm{kpt}} w_{i, \mathrm{opt}} w_{i, \text { escape }}$. It is important to note that $w_{i, \mathrm{kpt}}(\vec{k})$ is not necessarily equal to the number of other symmetry equivalent $k$ points in the first Brillouin zone (see Sec. IIE). In the following sections, we will derive an expression for each of these quantities.

\section{B. Derivation of photoexcitation probability}

According to the Spicer three-step model [12], photoemission first requires a photoexcitation from an initial valenceband state to a final conduction-band state. Since interband 
photoexcitations require conservation of crystal momentum, they can be either direct, where $\vec{k}_{\text {valence }}=\vec{k}_{\text {conduction }}$, or indirect through phonon coupling, where $\vec{k}_{\text {valence }}=\vec{k}_{\text {conduction }} \pm$ $\vec{k}_{\text {phonon. }}$ However, since indirect transitions depend on both an electron transition probability and a phonon transition probability, they are assumed to be less probable than direct transitions at low temperatures. As a result, we consider only contributions from direct optical transitions when computing photoemission probabilities.

Fermi's "golden rule" gives the probability of an optical transition per unit time between a conduction-band state, $\psi_{\vec{k}, c}(\vec{r})$, and a valence-band state, $\psi_{\vec{k}, v}(\vec{r})$, as

$$
\begin{aligned}
W(\vec{k}, \omega, T)= & \frac{2 \pi}{\hbar}\left\langle\psi_{\vec{k}, c}\left|H^{\prime}\right| \psi_{\vec{k}, v}\right\rangle^{2} \\
& \times \delta\left[E_{c}(\vec{k})-E_{v}(\vec{k})-\hbar \omega\right] F(T),
\end{aligned}
$$

where $H^{\prime}$ is the time-dependent electromagnetic perturbation on the system and $\delta$ is the Dirac delta function. The matrix elements, $\left\langle\psi_{\vec{k}, c}\left|H^{\prime}\right| \psi_{\vec{k}, v}\right\rangle$, incorporate the selection rules for the transitions between the initial and final states, whereas the $\delta$ function ensures conservation of energy between the photon and the states in the photocathode. To account for the occupancy differences in these states at finite temperatures $T$, we introduce an occupancy factor $F(T)$ :

$$
\begin{aligned}
F(T)= & f\left(E_{v}, T\right)\left[1-f\left(E_{c}, T\right)\right] \\
& -f\left(E_{c}, T\right)\left[1-f\left(E_{v}, T\right)\right] \\
= & f\left(E_{v}, T\right)-f\left(E_{c}, T\right),
\end{aligned}
$$

where $f(E, T)$ is the Fermi-Dirac distribution at an energy $E$ relative to the Fermi level. The temperature dependence is included by populating the Kohn-Sham DFT states at an electronic temperature $T$ with the occupancy of each state described by a Fermi-Dirac distribution. The first term in this expression represents the occupation of states available for photoexcitation from the valence state to the conduction state, while the second term represents the occupation of states available for stimulated emission from the conduction state to the valence state. Equation (10) is particularly important for modelling the photoemission in metals in order to accurately represent the photoexcitation probability of partially occupied bands close to the Fermi level.

The optical perturbation Hamiltonian $H^{\prime}$ in Fermi's golden rule is given by

$$
H^{\prime}=\frac{-e}{m c} \vec{A} \cdot \vec{p},
$$

where $\vec{A}$ is the vector potential of the optical field and $\vec{p}$ is the momentum operator. Since the wave vector of the light is small relative to the Brillouin-zone dimensions, the spatial dependence of $\vec{A}$ can be neglected and we can approximate the Hamiltonian matrix elements as

$$
\begin{aligned}
\left\langle\psi_{\vec{k}, c}\left|H^{\prime}\right| \psi_{\vec{k}, v}\right\rangle^{2} & =\left\langle\psi_{\vec{k}, c}\left|\frac{-e}{m c} \vec{A} \cdot \vec{p}\right| \psi_{\vec{k}, v}\right\rangle^{2} \\
& \approx\left(\frac{e}{m c}\right)^{2}|A|^{2}\left\langle\psi_{\vec{k}, c}|\vec{p}| \psi_{\vec{k}, v}\right\rangle^{2}
\end{aligned}
$$

Substituting the above Hamiltonian matrix elements into Fermi's golden rule gives

$$
\begin{aligned}
W(\vec{k}, \omega, T)= & \frac{2 \pi}{\hbar}\left(\frac{e}{m c}\right)^{2}|A|^{2}\left\langle\psi_{\vec{k}, c}|\vec{p}| \psi_{\vec{k}, v}\right\rangle^{2} \\
& \times \delta\left[E_{c}(\vec{k})-E_{v}(\vec{k})-\hbar \omega\right] \\
& \times\left[f\left(E_{v}, T\right)-f\left(E_{c}, T\right)\right] .
\end{aligned}
$$

For all $i$ pairs of conduction-band states and valence-band states we compute a relative photoexcitation probability weight, $w_{i, \mathrm{opt}}$, as

$$
\begin{aligned}
w_{i, \mathrm{opt}}(\vec{k}, \omega, T)= & \left\langle\psi_{\vec{k}, c}|\vec{p}| \psi_{\vec{k}, v}\right\rangle^{2} \\
& \times \delta\left[E_{c}(\vec{k})-E_{v}(\vec{k})-\hbar \omega\right] \\
& \times\left[f\left(E_{v}, T\right)-f\left(E_{c}, T\right)\right] .
\end{aligned}
$$

To evaluate $w_{i, \text { opt }}$ within the framework of DFT, we follow the methodology of Gajdoš et al. [21] and write the imaginary part of the frequency-dependent macroscopic dielectric function between a conduction-band state $c$ and a valence-band state $v$ in the independent particle approximation as

$$
\begin{aligned}
\varepsilon_{\alpha \beta}^{(2)}(\omega, \vec{k}) & \\
= & \frac{4 \pi^{2} e^{2}}{\Omega} \lim _{q \rightarrow 0}\left\langle u_{c+e_{\alpha} q}(\vec{k}) \mid u_{v}(\vec{k})\right\rangle \\
& \times\left\langle u_{c+e_{\beta} q}(\vec{k})\left|u_{v}(\vec{k})\right| \delta\left[E_{c}(\vec{k})-E_{v}(\vec{k})-\hbar \omega\right],\right.
\end{aligned}
$$

where $u_{c+e_{\alpha} q}(\vec{k})$ is the cell periodic part of a pseudowave function within the projector-augmented wave (PAW) methodology and $\Omega$ is the volume of the unit cell. The macroscopic dielectric function is evaluated at the $k$ point $\vec{k}$ perturbed by a small momentum $q$ in a Cartesian direction $e_{\alpha}$ corresponding to the direction of the polarization of the incident radiation. The macroscopic dielectric function in Eq. (15) can be related to Eq. (14) by substituting the momentum operator with the gradient operator and writing the cell periodic part of the Kohn-Sham wave functions as a first-order Taylor expansion. Since the frequency-dependent macroscopic dielectric function, $\varepsilon_{\alpha \beta}^{(2)}(\omega, \vec{k})$, is directly proportional to the absorption coefficient, we can relate the DFT calculated macroscopic dielectric function to the relative photoexcitation probability weight by

$$
\begin{aligned}
w_{i, \mathrm{opt}}= & \varepsilon_{\alpha \beta}^{(2)}(\omega, \vec{k}) \\
& \times\left[f\left(E_{v}, T\right)-f\left(E_{c}, T\right)\right] .
\end{aligned}
$$

Finally, we evaluate $\delta(x)$ in Eq. (15) as

$$
\delta(x)=\frac{1}{\sigma} \frac{e^{-x / \sigma}}{\left(1+e^{-x / \sigma}\right)^{2}},
$$

where $\sigma$ represents the lifetime broadening of the excited states. We assume a value of $\sigma=0.20 \mathrm{eV}$ for all calculations in this work (see Appendix A).

In this work, we calculate the intrinsic emittance for incident radiation that is polarized normal to the surface of the photocathode. However, the photoexcitation probability for any general incident angle can be calculated by substituting the relevant macroscopic dielectric function $\varepsilon_{\alpha \beta}^{(2)}$ into Eq. (16). 
The Dirac delta function weight given by Eq. (17) and the photoexcitation matrix elements, $\left\langle\psi_{\vec{k}, c}|\vec{p}| \psi_{\vec{k}, v}\right\rangle^{2} F(T)$, seen in Eq. (13) are plotted for $\mathrm{PbTe}(111)$ in Figs. 3(a) and 3(b), respectively.

\section{Electron scattering}

The second step in the Spicer three-step model [12] involves the migration of photoexcited electrons to the photocathode-vacuum interface, including electron scattering. In our model, we neglect the impacts of both inelastic and elastic electron scattering in the calculation of the intrinsic emittance. Whereas electron-electron scattering does not impact the calculated intrinsic emittance due to conservation of momentum, ignoring the impacts of electron-phonon scattering is equivalent to neglecting the energy dependence on the electron scattering mean free path as well as assuming that the electron scattering probability is isotropic with respect to the motion of the electron. The effects of these two assumptions have been calculated for copper and were found to cause an increase in the rms transverse momentum by $0.8 \%$ and $0.2 \%$, respectively, when using an experimentally determined electron-electron scattering length of $22 \AA$ [13]. More generally, we expect this approximation to best hold for materials with shorter skin depths, i.e., metals and more strongly absorbing semiconductors.

\section{Derivation of transmission probability}

Finally, the third step in the Spicer three-step model involves transmission of the electrons across the photocathodevacuum interface. From solving the Kohn-Sham equations, we obtain the Kohn-Sham states within the photocathode, $\psi_{\vec{k}_{\mathrm{in}}}(\vec{r})$, which can be expressed in terms of plane waves as

$$
\begin{aligned}
\psi_{\vec{k}_{\mathrm{in}}}(\vec{r})= & e^{i \vec{k}_{\mathrm{in}} \vec{r}} \sum_{\vec{G}} \alpha_{\vec{k}_{\mathrm{in}}}(\vec{G}) e^{i \vec{G} \vec{r}} \\
& +\operatorname{Re}^{i \vec{k}_{\mathrm{ref}} \vec{r}} \sum_{\vec{G}} \alpha_{\vec{k}_{\mathrm{ref}}}(\vec{G}) e^{i \vec{G} \vec{r}},
\end{aligned}
$$

where $\vec{k}_{\text {in }}=\left[k_{x, \text { in }}, k_{y, \text { in }}, k_{z, \text { in }}\right]$ is the crystal momentum wave vectors inside the photocathode, $\vec{k}_{\text {ref }}$ is the crystal momentum of internally reflected wave vectors, $\alpha_{\vec{k}}$ are the plane-wave expansion coefficients, $\vec{G}$ are the reciprocal-lattice vectors, and $R$ is the probability coefficient for the internally reflected wave function at the photocathode-vacuum interface.

At the photocathode-vacuum interface, we approximate the work function as a step potential with height $V_{0}$ located at $z=$ 0 . We then write the wave function of electrons transmitted across the photocathode-vacuum interface in terms of plane waves as

$$
\psi_{\vec{k}_{\text {out }}}(\vec{r})=T \sum_{\vec{k}_{\text {out }}} \alpha_{\vec{k}_{\text {out }}} e^{i \vec{k}_{\text {out }}}
$$

where $\vec{k}_{\text {out }}=\left[k_{x, \text { out }}, k_{y, \text { out }}, k_{z \text { out }}\right]$ and $T$ is the probability coefficient for the wave functions transmitted across the interface. If we represent the emitted electrons as free electrons, then (a)

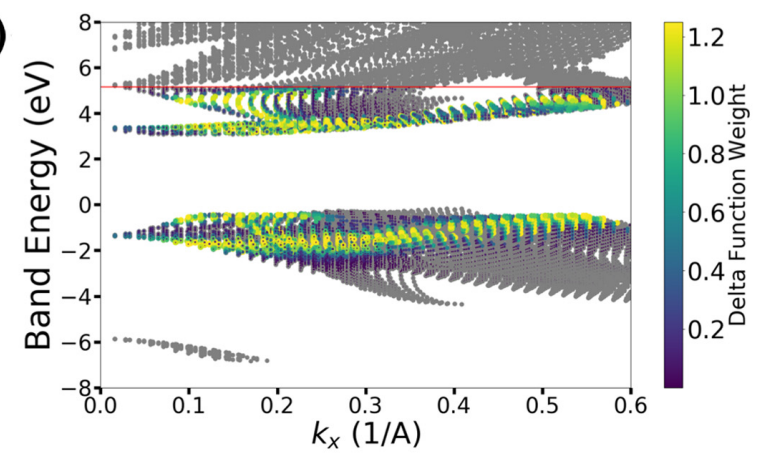

(b)

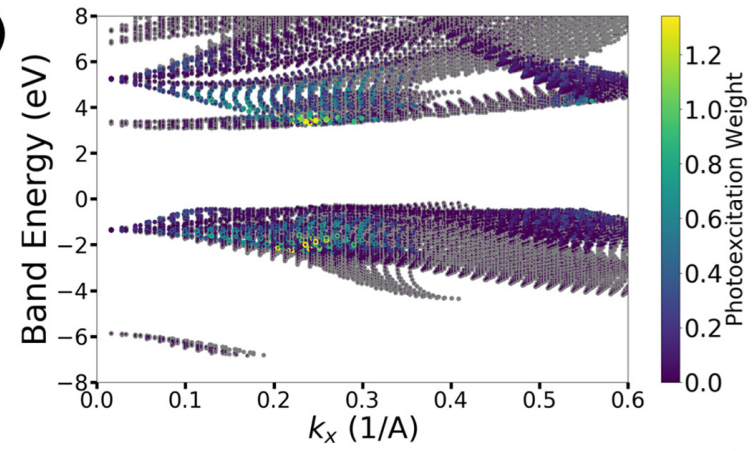

(c)

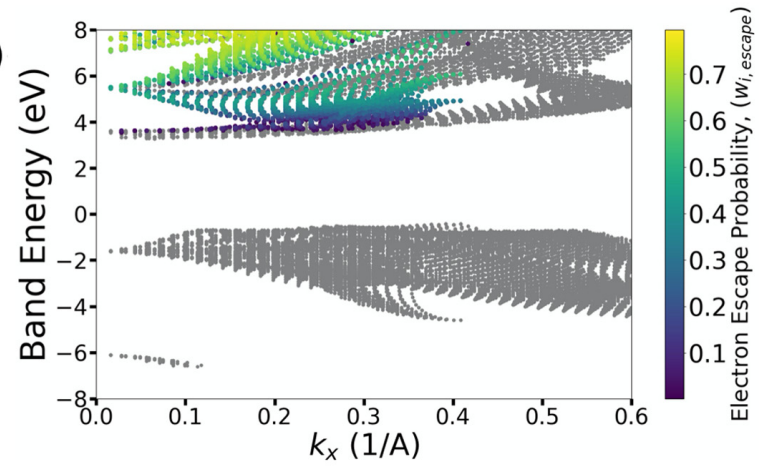

(d)

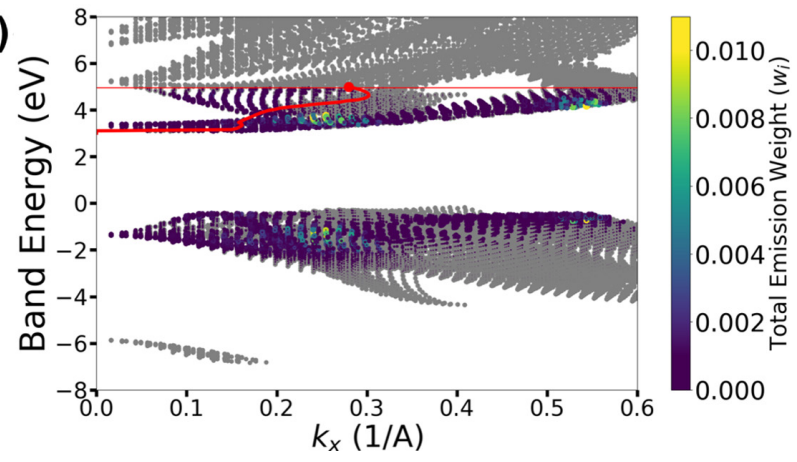

FIG. 3. Weights used in the calculation of the PbTe (111) intrinsic emittance: (a) Dirac delta function weight given by Eq. (17) at $\hbar \omega=5 \mathrm{eV}$, (b) the photoexcitation matrix elements, $\left\langle\psi_{\vec{k}, c}|\vec{p}| \psi_{\vec{k}, v}\right\rangle^{2} F(T)$, seen in Eq. (13), (c) the electron escape probability, $w_{i, \text { escape }}$, given by Eq. (30), and (d) the total electron emission weight $w_{i}$ given by Eq. (8) at $\hbar \omega=5 \mathrm{eV}$. The horizontal red lines in (a) and (d) correspond to the incident photon energy, $\hbar \omega=5 \mathrm{eV}$, whereas the red overlaid plot in (d) gives $\sqrt{\left\langle k_{x}^{2}\right\rangle}$ as a function of $\hbar \omega$. The conduction-band states below the vacuum level are omitted here for clarity. The plots shown here are for the PBE calculated $\mathrm{PbTe}$ states without fitting to the experimental band gap or electron effective mass. The weights in (d) are calculated as the product of the weights in plots (a)-(c). A video illustrating the incident photon energy dependence of (d) is given in the Supplemental Material [22]. 
$k_{z, \text { out }}$ is given by

$$
k_{z, \text { out }}=\sqrt{\frac{2 m}{\hbar^{2}}\left(E-V_{0}\right)-k_{x, \text { out }}^{2}-k_{y, \text { out }}^{2}},
$$

where $E$ is the energy of the photoexcited electron with respect to the Fermi level.

At the photocathode-vacuum interface, defined as $\vec{r}_{\text {int }}=$ $[x, y, 0]$, these wave functions must satisfy the boundary conditions, $\psi_{\vec{k}_{\text {in }}}\left(\vec{r}_{\text {int }}\right)=\psi_{\vec{k}_{\text {out }}}\left(\vec{r}_{\text {int }}\right)$ and $\frac{\partial}{\partial z} \psi_{\vec{k}_{\text {in }}}\left(\vec{r}_{\text {int }}\right)=\frac{\partial}{\partial z} \psi_{\vec{k}_{\text {out }}}\left(\vec{r}_{\text {int }}\right)$. Solving the first boundary condition at the interface and using $\vec{k}_{\text {in }} \cdot \vec{r}_{\text {int }}=\vec{k}_{\text {ref }} \cdot \vec{r}_{\text {int }}$ gives

$$
\begin{aligned}
& \sum_{G_{z}} \sum_{G_{x, y}}\left[\alpha_{\vec{k}_{\text {in }}}(\vec{G})+R \alpha_{\vec{k}_{\text {ref }}}(\vec{G})\right] e^{i\left(\vec{k}_{\text {in }}+\vec{G}\right) \vec{r}_{\text {int }}} \\
& \quad=T \sum_{k_{x, \text { out }}} \sum_{k_{y, \text { out }}} \alpha_{\vec{k}_{\text {out }}} e^{i \vec{k}_{\text {out }} \vec{r}_{\text {int }}} .
\end{aligned}
$$

Eq. (21) then gives the conditions

$$
\begin{aligned}
& k_{x, \text { in }}+G_{x}=k_{x, \text { out }}, \\
& k_{y, \text { in }}+G_{y}=k_{y, \text { out }} .
\end{aligned}
$$

Substituting these boundary conditions into Eq. (21) gives the condition for every value of $G_{x}$ and $G_{y}$ :

$$
\sum_{G_{z}} \alpha_{\vec{k}_{\mathrm{in}}}(\vec{G})+R \alpha_{\vec{k}_{\mathrm{ref}}}(\vec{G})=T \alpha_{\vec{k}_{\mathrm{out}}} .
$$

Now, solving for the boundary condition, $\frac{\partial}{\partial z} \psi_{\vec{k}_{\text {in }}}\left(\vec{r}_{\text {int }}\right)=$ $\frac{\partial}{\partial z} \psi_{\vec{k}_{\text {out }}}\left(\vec{r}_{\text {int }}\right)$, gives

$$
\begin{aligned}
& \sum_{\vec{G}} i\left(G_{z}+k_{z, \text { in }}\right) e^{i \vec{G} \vec{r}_{\text {int }}} e^{i \vec{k}_{\text {in }} \vec{r}_{\text {int }}} \alpha_{\vec{k}_{\text {in }}}(\vec{G}) \\
& +i\left(G_{z}+k_{z, \text { ref }}\right) R e^{i \vec{G} \vec{r}_{\text {int }}} e^{i \vec{k}_{\text {ref }} \vec{r}_{\text {int }}} \alpha_{\vec{k}_{\text {ref }}}(\vec{G}) \\
& -T \sum_{\vec{k}_{\text {out }}} i k_{z, \text { out }} e^{i \vec{k}_{\text {out }} \vec{r}_{\text {int }}} \alpha_{\vec{k}_{\text {out }}}=0 .
\end{aligned}
$$

Equations (22) and (23) can then be substituted into Eq. (25) to give for every value of $G_{x}$ and $G_{y}$ :

$$
\begin{aligned}
& \sum_{G_{z}}\left(G_{z}+k_{z, \text { in }}\right) \alpha_{\vec{k}_{\text {in }}}(\vec{G})+\left(G_{z}+k_{z, \text { ref }}\right) R \alpha_{\vec{k}_{\text {ref }}}(\vec{G}) \\
& \quad-T k_{z, \text { out }} \alpha_{\vec{k}_{\text {out }}}=0 .
\end{aligned}
$$

The transmission probability across the photocathode-vacuum interface is given by

$$
\frac{\left(\frac{\partial}{\partial z} \psi_{\vec{k}_{\text {out }}}\left(\vec{r}_{\text {int }}\right)\right) \psi_{\vec{k}_{\text {out }}}^{*}\left(\vec{r}_{\text {int }}\right)-c . c .}{\left(\frac{\partial}{\partial z} \psi_{\vec{k}_{\text {inc. }}}\left(\vec{r}_{\text {int }}\right)\right) \psi_{\vec{k}_{\text {inc. }}^{*}}^{*}\left(\vec{r}_{\text {int }}\right)-c . c .},
$$

where $c . c$. is the complex conjugate of the preceding term and $\psi_{\vec{k}_{\text {inc. }}}(\vec{r})$ is the incident wave function, given by $\psi_{\vec{k}_{\text {inc. }}}(\vec{r})=$ $\sum_{\vec{G}} \alpha_{\vec{k}_{\text {in }}}(\vec{G}) e^{i\left(\vec{k}_{\text {in }}+\vec{G}\right) \vec{r}}$. The first term of the numerator of
Eq. (27) can be evaluated to give

$$
\begin{aligned}
& T^{2} \sum_{\vec{k}_{\text {out }}} i k_{z, \text { out }} \alpha_{\vec{k}_{\text {out }}} e^{i \vec{k}_{\text {out }} \vec{r}_{\text {int }}} \\
& \quad \times \sum_{\vec{k}_{\text {out }}^{\prime}} \alpha_{\vec{k}_{\text {out }}^{\prime}}^{*} e^{-i \vec{k}_{\text {out }}^{\prime} \vec{r}_{\text {int }}} \\
& =T^{2} \sum_{\vec{k}_{\text {out }}} \sum_{\vec{k}_{\text {out }}^{\prime}} i k_{z, \text { out }} \alpha_{\vec{k}_{\text {out }}} \alpha_{\vec{k}_{\text {out }}^{\prime}}^{*} e^{i\left(\vec{k}_{\text {out }}-\vec{k}_{\text {out }}^{\prime}\right) \vec{r}_{\text {int }}}
\end{aligned}
$$

Similarly, the first term of the denominator of Eq. (27) can be evaluated to give

$$
\sum_{\vec{G}} \sum_{\vec{G}^{\prime}} i\left(k_{z, \text { in }}+G_{z}\right) \times \alpha_{\vec{k}_{\text {in }}}(\vec{G}) \alpha_{\vec{k}_{\text {in }}}^{*}\left(\vec{G}^{\prime}\right) e^{i\left(\vec{G}-\vec{G}^{\prime}\right) \vec{r}_{\text {int }}} .
$$

Notably, if we only consider $\vec{G}=\vec{G}^{\prime}=\overrightarrow{0}$ components of the incident Bloch state to contribute to the photoemission and substitute in Eqs. (24) and (26), the transmission probability is given by

$$
\begin{gathered}
\frac{2 T^{2} i k_{z, \text { out }} \alpha_{\vec{k}_{\text {out }}} \alpha_{\vec{k}_{\text {out }}^{\prime}}^{*}}{2 i k_{z, \text { in }} \alpha_{\vec{k}_{\text {in }}}(\overrightarrow{0}) \alpha_{\vec{k}_{\text {in }}^{*}}^{*}(\overrightarrow{0})}=\frac{k_{z, \text { out }}}{k_{z, \text { in }}}\left(\frac{2 k_{z, \text { in }}}{k_{z, \text { out }}+k_{z, \text { in }}}\right)^{2} \\
=\frac{4 k_{z, \text { in }} k_{z, \text { out }}}{\left(k_{z, \text { out }}+k_{z, \text { in }}\right)^{2}}=w_{i, \text { scape }}(\vec{k}),
\end{gathered}
$$

which is equivalent to the electron transmission probability across a one-dimensional potential step. However, Eq. (30) is strictly applicable only in cases where $\vec{G}=\vec{G}^{\prime}=\overrightarrow{0}$ components of the Bloch waves are nonzero and $k_{z, \text { ref }}=-k_{z \text {,in }}$. In this case, there is only one value of $\vec{k}_{\text {out }}$ that satisfies $k_{x, \text { out }}=k_{x, \text { in }}$ and $k_{y, \text { out }}=k_{y, \text { in }}$.

In general, $G_{x, y} \neq 0$ terms in the Bloch states can lead to photoemission at more than one value of $\vec{k}_{\text {out }}$ if they satisfy

$$
\frac{2 m}{\hbar^{2}}\left(E-V_{0}\right)>\left(k_{x, \text { in }}+\frac{2 \pi n}{a}\right)^{2}+\left(k_{y, \text { in }}+\frac{2 \pi l}{b}\right)^{2},
$$

where $a$ and $b$ are the real-space lattice vectors that span the transverse surface of the photocathode material and $n$ and $l$ are integers. This constraint on $\vec{G}$ ensures that the emitted electrons have real $k_{z \text {,out }}$ in Eq. (20), which is required to give a real transmission probability [Eq. (30)].

The values of $\vec{G}$ which satisfy the constraint given by Eq. (31) are plotted in Appendix B. For Rh(110), the lowest excess energy that satisfies Eq. (31) is $2.54 \mathrm{eV}$, which is above the experimentally relevant excess energies. For all intrinsic emittance calculations in this work, we take emitted electron energies to be sufficiently small that Eq. (31) holds for $n=0$ and $l=0$. In this case, there is a single beam of electrons emitted.

The full Bloch solution for escape probability can be obtained by combining Eqs. (24), (26), (28), and (29) assuming that the crystal/vacuum interface is perfectly sharp on subatomic scales. In this work, we take Eq. (30) as an approximation of $w_{i \text {,escape }}$ in consideration of more gradual surface potential variation.

The wave vectors inside the material, $\vec{k}_{\text {in }}$, used in the calculation of the transmission probability are obtained directly from the $k$ points of the DFT calculation. For each pair of 
states with wave vector, $\vec{k}_{\text {in }}$, obtained in Sec. II A, Eq. (30) allows us to determine the probability of emitting an electron with wave vector $\vec{k}_{\text {out }}$. We note that this approach directly incorporates the vacuum density of states and evanescent states [Eq. (20)] into the calculation of the transmission co-

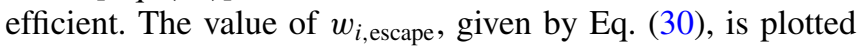
for $\mathrm{PbTe}(111)$ in Fig. 3(c).

\section{E. DFT details}

The Vienna Ab initio Simulation Package (VASP 5.4.1) [23] was used to perform all Kohn-Sham density functional theory (KS-DFT) [19,20] computations with the projector augmented-wave (PAW) method [24]. All photocathode structures were originally obtained from the Materials Project database [25] and were further relaxed to an ionic step energy convergence of $10^{-4} \mathrm{eV}$ using self-consistent, periodic DFT. For surface calculations, we create a slab of at least five atomic layers to ensure that the surface energy is converged with respect to the slab thickness. We also include a vacuum space of at least $15 \AA$ between periodic slabs to preclude interactions between periodic images. The atoms in the slabs were fixed to their bulk atomic positions and were not allowed to relax. For both bulk and surface calculations, we use Miller indices to denote the surface from which the electrons are being emitted. In terms of our photoemission model, this designation only affects the value of the work function used in calculating the transmission probability and the transverse direction. As an illustrative example, the intrinsic emittance calculations shown here for bulk $\operatorname{PbTe}(111)$ are calculated assuming photoexcitations occur only between bulk PbTe states, that are then transported through the crystal and emitted through the (111) surface of PbTe.

Obtaining the properties of a periodic system generally involves integrating over all $k$ points in the first Brillouin zone. Computationally, these integrals are evaluated by performing a weighted sum over a dense mesh of $k$ points. For this purpose, we use a uniform $k$-point mesh of at least 10000 $k$ points per reciprocal atom to sample the $k_{z} \geqslant 0$ half of the first Brillouin zone. A weight of $w_{i, \mathrm{kpt}}$ is given to each $k$ point in the symmetry irreducible Brillouin zone, representing the number of other equivalent $k$ points. We consider two $k$ points to be equivalent if they are related by a symmetry operation of the crystal's symmetry group and also have the same scalar projection onto the transverse or $x$ axis, $k_{x}^{2}$. Meeting both of these symmetry conditions ensures that the total emission weight, $w_{i}(\vec{k}, \omega, T)$, and the magnitude of $k_{x}^{2}$ at the two $k$ points are identical and both $k$ points will provide equal contributions to the intrinsic emittance. Nonunique $k$ points can then be removed until only unique $k$ points remain in the Brillouin zone. This approach ensures that non-high-symmetry $k$ points are included in the distribution of emitted electrons. The component of $\vec{k}$ in any reciprocal space direction $\vec{a}$ can then be determined from the scalar projection of $\vec{k}$ onto $\vec{a}$. As such, we calculate the scalar projection of all $k$ points onto the $x$ axis to calculate $k_{x}^{2}$.

All DFT calculations in this work represent the wave function as an expansion of a plane-wave basis set. To ensure convergence with respect to the size of the basis set, this expansion is chosen to include all reciprocal-lattice vectors up to a predetermined kinetic-energy cutoff. We use a kineticenergy cutoff of $300 \mathrm{eV}$ for Rh, W, and Mo, $320 \mathrm{eV}$ for $\mathrm{PbTe}$, and $520 \mathrm{eV}$ for $\mathrm{Cu}$. The number of bands included in each calculation is set to ensure that the highest energy conduction-band state at every $k$ point is at least $5 \mathrm{eV}$ above the vacuum level.

The exchange-correlation energy is treated with the generalized gradient approximation (GGA) PBE functional for all calculations [26]. However, the Perdew-Burke-Ernzerhof (PBE) functional is known to generally underestimate the quasiparticle band gap of semiconductors. For the calculation of the intrinsic emittance of photocathodes, this often causes a discrepancy between the calculated and experimental onset of photoemission. With the $G W$ approximation of manybody perturbation theory, the quasiparticle bands of semiconducting photocathode materials can be more accurately predicted without relying on experimental parametrization $[27,28]$. However, most of the commonly used materials databases only provide PBE calculated electronic structures. Therefore, we choose to assess the performance of our model with the PBE functional in order to illustrate the accuracy that can be expected if using band structures from materials databases.

To account for the errors in the PBE calculated band structure, we shift the PBE calculated bands to match the experimentally measured band gap. For PbTe, the PBE calculated band gap is $0.80 \mathrm{eV}$ with the Fermi level located at the valence-band maximum. However, optical absorption measurements of pure, single-crystal $\mathrm{PbTe}$ have determined the optical band gap to be $0.19 \mathrm{eV}$ at low temperatures, with the Fermi level located at the conduction-band minimum [29,30]. To match this experimental band structure, we shift the PBE calculated conduction bands downwards by $0.80 \mathrm{eV}$ and the PBE calculated valence bands downwards by $0.19 \mathrm{eV}$. After shifting the bands, we also scale the PBE calculated $\mathrm{PbTe}$ conduction bands to match the experimentally measured $\mathrm{PbTe}$ electron effective mass. This is performed by scaling the energy of the conduction bands by the ratio of the DFT calculated electron effective mass of $0.15 m_{e}$ to the experimentally determined electron effective mass of $0.24 m_{e}$ [31].

In the case of both semiconducting and metallic photocathodes, an experimentally determined work function is used to determine the lowest-lying conduction-band states which can still give rise to photoemission. We find that the intrinsic emittance at a given photon energy is relatively independent on the value of the work function for photon energies more than approximately $1 \mathrm{eV}$ higher than the work function (see Fig. 6).

\section{MODEL PERFORMANCE}

To gain some intuition into how the photoemission process is represented by our model, we calculate the intrinsic emittance of bulk PbTe(111) at $300 \mathrm{~K}$ with various different representations of $w_{i}$, shown in Fig. 4. By assigning various values to $w_{i}$, we can explore the significance of each step in the photoemission process to the calculated intrinsic emittance. We chose to perform this test of our method on PbTe(111) because the intrinsic emittance measured as a function of 


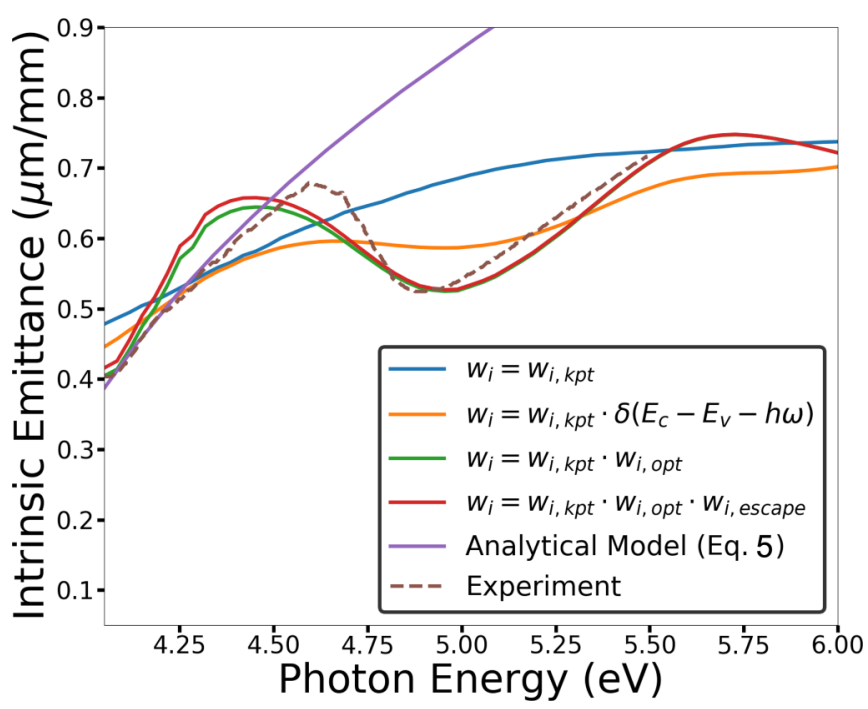

FIG. 4. Bulk PbTe(111) intrinsic emittance at $300 \mathrm{~K}$ calculated with various different total emission probabilities $w_{i}$. The orange line exhibits a nonmonotonic relationship between intrinsic emittance and energy, indicating that the delta function, $\delta\left[E_{c}(\vec{k})-E_{v}(\vec{k})-\hbar \omega\right]$, and optical matrix elements both give rise to the nonmonotonic behavior. Reported experimental measurements are also shown for comparison [10].

photon energy has been reported to display an interesting nonmonotonic behavior, shown in Fig. 4 [10]. This behavior cannot be captured by Eq. (5) $\left(\varepsilon_{\text {int }}=\sqrt{\frac{\hbar \omega-\phi}{3 m c^{2}}}\right)$ which predicts the intrinsic emittance is a monotonically increasing function of photon energy.

We first calculate the intrinsic emittance of $\mathrm{PbTe}(111)$ with constant $w_{i, \text { opt }}$ and $w_{i \text {,escape }}$, which is equivalent to assuming that all pairs of initial and final states, $\psi_{\vec{k}, v}(\vec{r})$ and $\psi_{\vec{k}, c}(\vec{r})$, contribute equally to the photoemission, given that the photon is energetic enough to induce the transition (blue line in Fig. 4). This approach does display the correct behavior at low photon energies, but fails to capture the experimentally observed peak at $\hbar \omega=4.6 \mathrm{eV}$. This behavior is expected with constant $w_{i, \text { opt }}$, since assigning an equal contribution to all possible optical transitions results in a monotonically increasing calculated intrinsic emittance as higher $k_{x}$ states become energetically accessible.

The effects of the electron transmission probability $w_{i \text {,escape }}$ are illustrated by comparing the green and red lines in Fig. 4. The inclusion of this probability seems to have minimal qualitative impact on the calculated intrinsic emittance for $\mathrm{PbTe}(111)$. This is in part due to the initial removal of all conduction-band states located below the vacuum level.

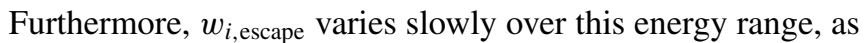
shown in Fig. 3(c). For example, conduction states located 0.5 and $0.8 \mathrm{eV}$ above the vacuum level in a photocathode with a work function of $4 \mathrm{eV}$ have transmission probabilities of $75 \%$ and $82 \%$, respectively. Although $w_{i \text {,escape seems to have }}$ a minimal impact on the calculated results, this is unlikely to be true in general. However, it is computationally inexpensive to calculate $w_{i, \text { escape }}$ and is essential for developing a generally applicable photoemission model.

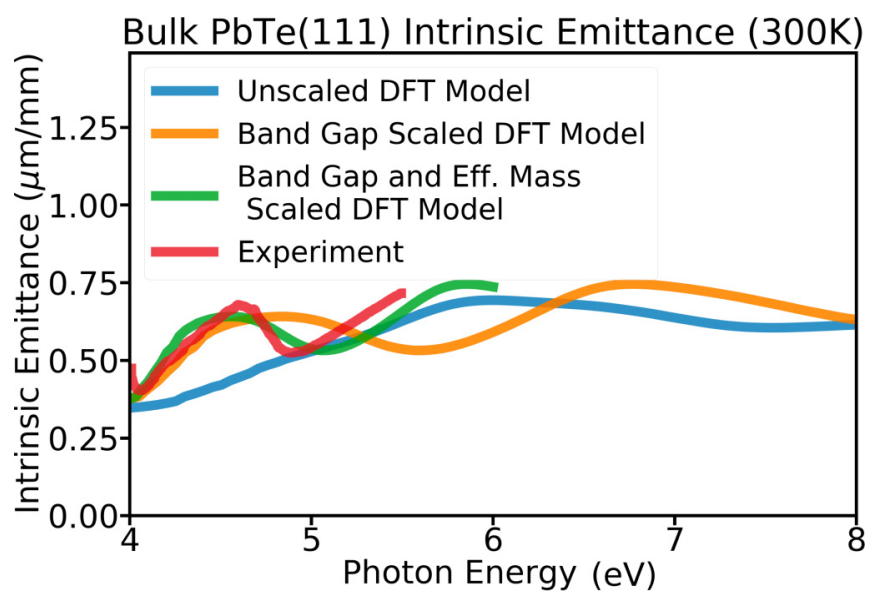

FIG. 5. Intrinsic emittance of bulk $\mathrm{PbTe}(111)$ at $300 \mathrm{~K}$ calculated without any experimental scaling factors (blue), shifting the DFT calculated bands to match the experimental band gap of $0.19 \mathrm{eV}$ [29] (orange), and fitting the bands to match the experimental band gap and electron effective mass of $0.24 m_{e}$ (green) [31]. The reported experimental measurements of intrinsic emittance are also shown for comparison (red) [10].

The experimentally observed peak at $\hbar \omega=4.6 \mathrm{eV}$ appears in our calculated results only upon inclusion of the Dirac delta function and optical matrix element portions of $w_{i \text {,opt }}$, which assigns greater contributions to only the states which satisfy $E_{c}(\vec{k})-E_{v}(\vec{k}) \cong \hbar \omega$. The observed decreases in the intrinsic emittance with increasing photon energy can then be explained by the emergence of optically active, low transverse momentum states that are only accessible at sufficiently

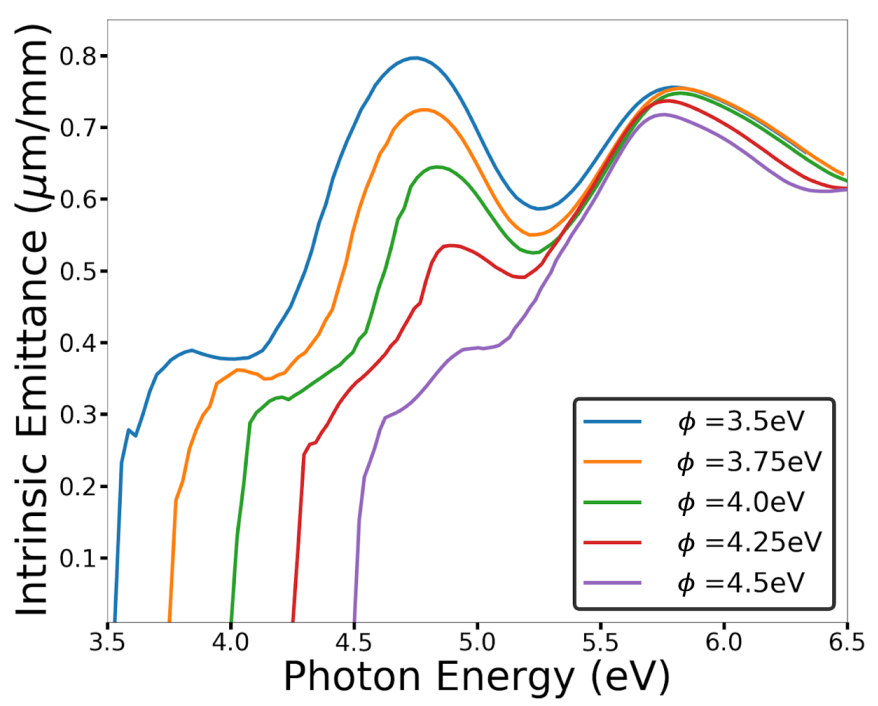

FIG. 6. Calculated variation in the intrinsic emittance of bulk $\mathrm{PbTe}(111)$ with respect to the work function $\phi$. The precise value of the work function has considerable impact on the calculated emittance at the photoemission threshold, but is less significant at high photon energies. The experimentally measured work function of $\mathrm{PbTe}(111)$ is reported to be $4.0 \mathrm{eV}$ [10] whereas the LDA calculated work function is reported to be 4.21 and $4.54 \mathrm{eV}$ for $\mathrm{Pb}$ and $\mathrm{Te}$ terminated surfaces, respectively [18]. 


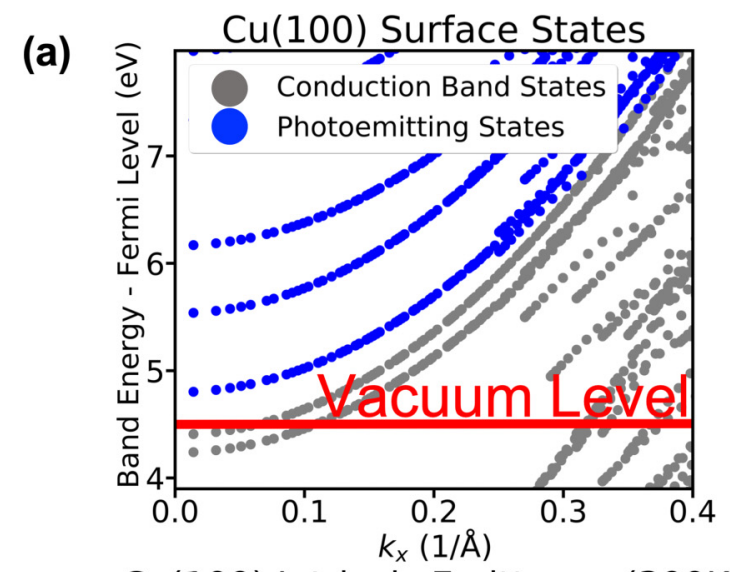

(c)

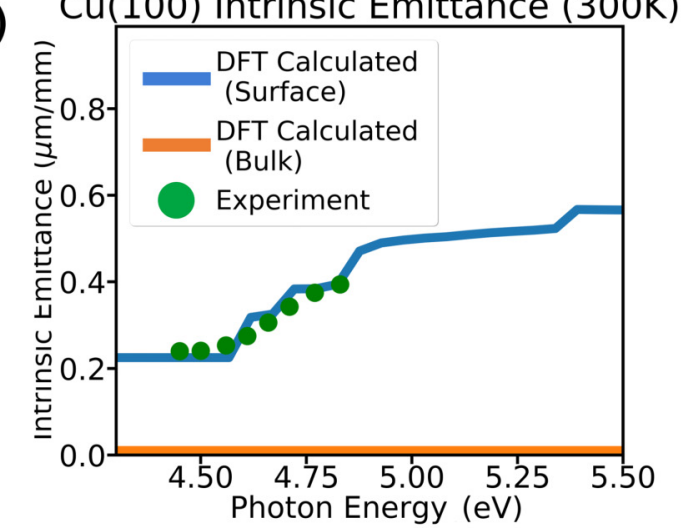

(b)

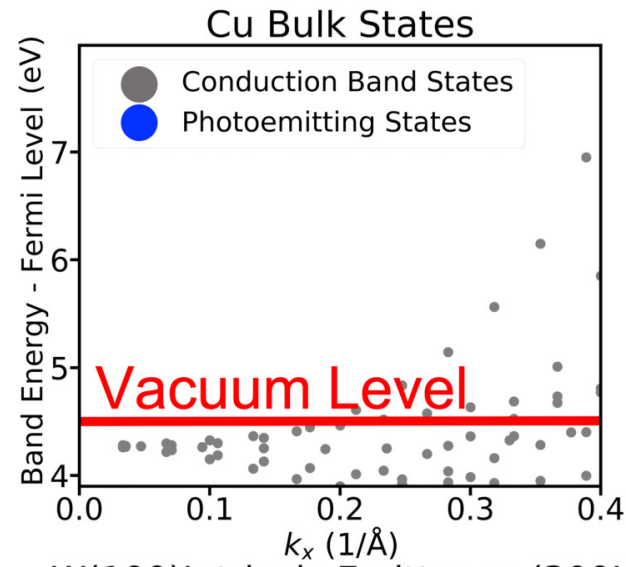

(d)

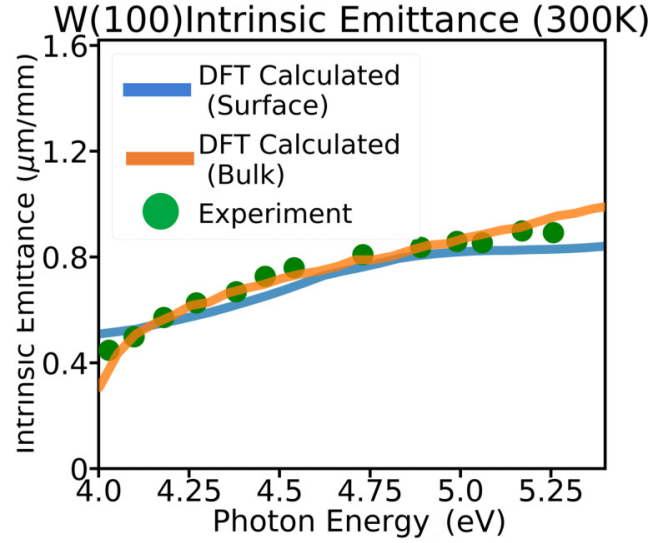

FIG. 7. Effect of surface states on intrinsic emittance calculations. DFT calculated conduction-band states for a (a) Cu(100) surface slab and (b) bulk $\mathrm{Cu}$. The conduction-band states which can contribute to the photoemission are highlighted in blue $\left(w_{i} \neq 0\right.$ at $\left.\hbar \omega \geqslant 5 \mathrm{eV}\right)$. (c) DFT calculated and experimental intrinsic emittance of $\mathrm{Cu}(100)$ at $300 \mathrm{~K}$ [32]. The experimentally observed low emittance at the photoemission threshold in $\mathrm{Cu}(100)$ must arise from surface states, due to the absence of bulk states directly above the vacuum level. (d) The experimentally measured intrinsic emittance for $\mathrm{W}(100)$ [33] is reasonably well described by both bulk and surface slab calculations, in contrast to Cu(100).

high photon energies (see the Supplemental Material [22]). Quantitatively, the best agreement with experiment is obtained when we describe the full photoexcitation probability with Eq. (8), illustrating the importance of including all of the aforementioned steps in the photoemission process.

Figure 5 illustrates the performance of our model without scaling the calculated conduction bands to match the experimentally measured effective mass and band gap. We calculate the PBE band gap of PbTe to be $0.82 \mathrm{eV}$ and electron effective mass to be $0.15 m_{e}$, whereas the experimentally measured values are $0.19 \mathrm{eV}$ and $0.24 m_{e}$, respectively [29,31]. In this work, we choose to benchmark the performance of our model with these experimental parameters included to illustrate how well our model describes the photoemission process itself, rather than how well semilocal DFT describes the electronic structure of the material. In other words, we strive for the errors reported in this work to represent the error if the exact electronic structure is known. However, in materials without experimental characterization, the accuracy of DFT predictions of the intrinsic emittance will be impacted by the inaccuracies of the chosen DFT functional. As illustrated in Fig. 5, semilocal DFT accurately predicts the magnitude and nonmonotonic behavior of the intrinsic emittance without any experimental fitting of the band structure. However, the relationship between the intrinsic emittance and incident photon energy is misaligned due to the overestimation of the GGA band gap by $0.6 \mathrm{eV}$, compared to experimental measurements [29].

Although considerable effort has been placed in accurately measuring the work function of photocathodes, the measured values of work function can vary by up to $\pm 1 \mathrm{eV}$, as a result of the measurement technique, temperature, sample geometry, and the presence of contaminants on the photocathode surface $[34,35]$. Since our method uses experimental work-function values to determine which conduction-band states are energetic enough to permit electron emission, it is essential to test the robustness of our model with respect to these variations in measured work-function values. We choose to use experimental work-function values in our model to allow for a direct comparison of the intrinsic emittance predicted by our model with the experimentally measured emittance. However, PBE calculated work functions of elemental crystals were previously found to agree with experimental values with an average error of $0.30 \mathrm{eV}$, which is comparable to the experimental precision [36]. Therefore, we expect the performance of our model would not be significantly impacted by the use of DFT calculated work functions, rather than experimentally obtained work functions.

Figure 6 depicts the calculated intrinsic emittance of bulk $\mathrm{PbTe}(111)$ for different values of work function. In the limit of $\hbar \omega \gg \phi$, the calculated intrinsic emittance is relatively insensitive to the value of the work function. However, when 
$\hbar \omega \approx \phi$, the calculated intrinsic emittance is significantly impacted by the choice of $\phi$. This is expected since the work function determines the minimum energy required by optical transitions to contribute to photoemission. When $\hbar \omega \approx$ $\phi$, the distribution of photoexcited electrons in $k$ space is restricted to the narrow range of conduction states which satisfy $E_{c}(\vec{k}) \approx E_{\text {vacuum. }}$. Given the high variation in possible work-function values, we can expect to be more confident in our model's predictions at higher photon energies than at lower photon energies. However, the variation of intrinsic emittance with work function hints at the exciting possibility of engineering low intrinsic emittance photocathodes through precisely controlling the work function, such that only states with low transverse momentum contribute to photoemission.

The impact of including surface states in our calculations is depicted in Fig. 7. Experimentally, $\mathrm{Cu}(100)$ exhibits low intrinsic emittance at the photoemission threshold, which is depicted by Fig. 7(c) [32]. However, the bulk conduction bands calculated by DFT show an absence of photoemitting conduction-band states until $\sim 7 \mathrm{eV}$ above the Fermi level, precluding low intrinsic emittance in bulk $\mathrm{Cu}$ for photon energies below $7 \mathrm{eV}$. In comparison, the DFT-calculated surface conduction bands show low transverse momentum states directly above the vacuum level, in agreement with the experimental measurements seen in Fig. 7(c). As such, this example provides direct evidence for the necessity of including surface states to accurately describe the photoemission in some materials.

However, in other materials such as W(100), agreement with the experimentally measured intrinsic emittance can be obtained by including the bulk states only [Fig. 7(d)]. Surprisingly, slightly better agreement with the experimentally measured intrinsic emittance is obtained when using a bulk $\mathrm{W}$ simulation cell instead of a surface slab. This may in part be due to our model assuming that all states are receiving the same intensity of photons from the incident light source. However, the intensity of light propagating through the photocathode material will be attenuated by a factor of $1 / e$ once it travels a distance known as the skin depth. Therefore, we might expect that the photoemission calculated with our model will be better described by bulk states for materials with large skin depths such as semiconductors.

The skin depth also plays an important role in the transport of electrons to the surface of the photocathode. For an electron located a distance $s$ beneath the photocathode surface, the probability of reaching the surface without experiencing electron-electron scattering, $F_{e-e}(\omega)$, is given by [13]

$$
F_{e-e}(\omega)=\frac{1}{1+\frac{\lambda_{\text {opt }}(\omega)}{\lambda_{e-e}}},
$$

where $\frac{\lambda_{\mathrm{opp}}(\omega)}{\lambda_{e-e}}$ is the ratio of the optical absorption depth to the electron-electron scattering mean free path. For materials with small $\lambda_{\text {opt }}(\omega)$, the majority of electrons that contribute to photoemission must be located near the surface. For $\mathrm{Cu}$, the DFT calculated skin depth has previously been reported to be $400 \AA$ at a photon energy of $5 \mathrm{eV}$, whereas the skin depth of bulk W is $250 \AA$ at a photon energy of $5 \mathrm{eV}$ [37].
For comparison, the average escape length of semiconducting GaAs is $6 \times 10^{4} \AA$ at a photon energy of approximately $2 \mathrm{eV}$ [38], suggesting the photoemission of semiconductors to be well described by bulk states. Based on these skin depths, one might expect the photoemission of $\mathrm{Cu}$ to be better described by bulk states than $\mathrm{W}$, in opposition to what is shown in Fig. 7. However, due to the absence of photoemitting bulk $\mathrm{Cu}$ states, the experimentally observed photoemission threshold at $4.5 \mathrm{eV}$ in $\mathrm{Cu}(100)$ must arise from surface states. Generally, we expect that materials with small skin depths will require calculations of both the bulk and surface states to consistently model the photoemission. Recently, a layer-by-layer decomposition approach has been reported to account for the differing light intensity incident on bulk and surface states [39].

To test how well our model can generalize to a wide range of materials, we calculate the intrinsic emittance for five candidate materials $[\mathrm{Cu}(100), \mathrm{Rh}(110), \mathrm{PbTe}(111), \mathrm{Mo}(100)$, and W(100)] (Fig. 8). This list of materials includes two facecentered-cubic (fcc) metals ( $\mathrm{Cu}$ and $\mathrm{Rh}$ ), two body-centeredcubic (bcc) metals (Mo and $\mathrm{W}$ ), and a rocksalt structure semiconductor $(\mathrm{PbTe})$. We also calculate the mean absolute error (MAE) between our model and the experimental measurements and compare this to the MAE of the analytical expression given in Eq. (5) (Fig. 9). We find that the analytical expression performs remarkably well for $\mathrm{Cu}, \mathrm{Mo}$, and $\mathrm{W}$. Since Eq. (5) assumes a free-electron model and constant density of states, it works well for these elemental metals where these assumptions are more valid than for semiconductors and more complex metals. Furthermore, we expect the optical transition probability to have a larger impact on the photoemission in materials with complex band structures (such as $\mathrm{PbTe}$ ), rather than elemental metals (such as $\mathrm{Cu}$ ). This may allow the analytical model to accurately describe the photoemission of simple metals, but prevents it from generalizing well to all materials.

The analytical expression qualitatively fails to describe the photoemission in $\mathrm{PbTe}(111)$ with a MAE of $0.270 \mu \mathrm{m} / \mathrm{mm}$, whereas our model achieves a MAE of $0.054 \mu \mathrm{m} / \mathrm{mm}$ (Fig. 9). This performance is quite noteworthy in light of previous modeling efforts on $\mathrm{PbTe}(111)$. A previous DFT-based photoemission analysis [18] predicted the mean transverse energy of $\mathrm{PbTe}(111)$ to be $20 \times$ smaller than experimental measurements [10]. Since this previous DFT-based analysis represented the electronic structure of PbTe with the electron's effective mass, this result may highlight the limitations of using effective mass to fully capture the complexity of the photocathode's electronic structure.

Across the five materials shown in Fig. 9, our model achieved an average MAE of $0.044 \mu \mathrm{m} / \mathrm{mm}$, with a maximum MAE of $0.058 \mu \mathrm{m} / \mathrm{mm}$. The analytical expression achieved an average MAE of $0.088 \mu \mathrm{m} / \mathrm{mm}$, with a maximum MAE of $0.270 \mu \mathrm{m} / \mathrm{mm}$. While our model performs slightly better on average than the analytical expression, we note that our model is considerably more robust at handling a wider range of materials. This distinction becomes increasingly important when attempting to predict the photoemission properties of candidate photocathodes for which experimental data have not been obtained. Whereas the analytical expression fails to accurately predict the intrinsic emittance of $\mathrm{Rh}$ and $\mathrm{PbTe}$, 

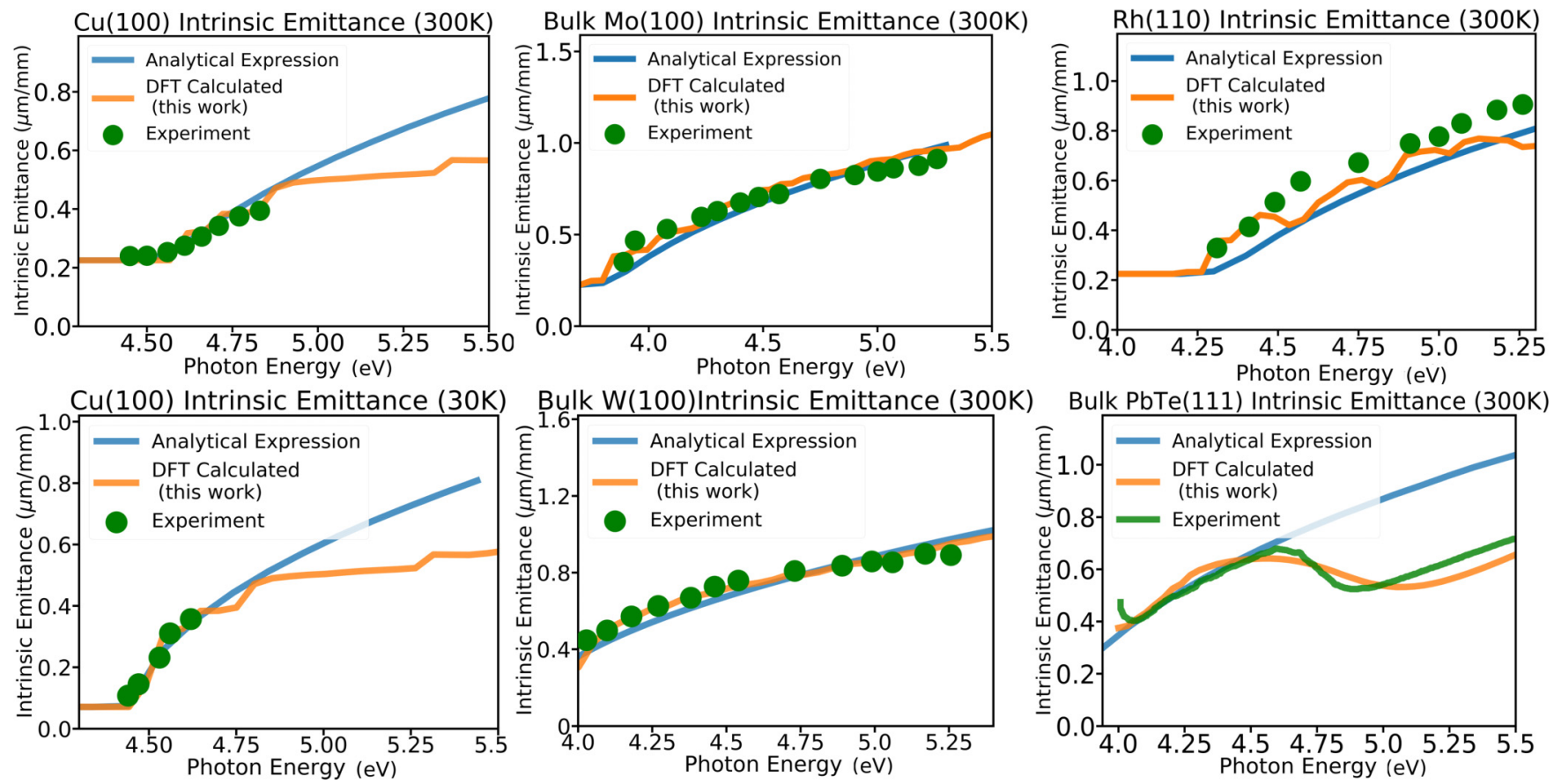

FIG. 8. Intrinsic emittance for five candidate materials [Cu(100), $\mathrm{Rh}(110), \mathrm{PbTe}(111), \mathrm{Mo}(100)$, and $\mathrm{W}(100)]$. We compare the analytical expression shown in Eq. (5), our DFT model, and previous experimental measurements [10,32,33,40]. The Rh, Mo, and W measurements were obtained with a solenoid scan technique [40], whereas the $\mathrm{Cu}$ and PbTe measurements were obtained using a free-flight method [32]. We calculated the intrinsic emittance of $\mathrm{Cu}(100)$ and $\mathrm{Rh}(110)$ from their surface bands, whereas we calculated the intrinsic emittance of W(100), $\mathrm{Mo}(100)$, and $\mathrm{PbTe}(111)$ from their bulk bands. Experimentally, $\mathrm{Rh}(110)$ was observed to have a surface oxide layer during the intrinsic emittance measurements, whereas $\mathrm{Cu}(100)$ was measured with an atomically flat and clean surface. The ability of our model to also account for finite-temperature effects is also illustrated for $\mathrm{Cu}(100)$ at 300 and $30 \mathrm{~K}$.

the MAE of our model is comparable to the experimental precision for all materials tested (Fig. 9), demonstrating the utility of our model as a tool for engineering new photocathode materials. Furthermore, since the analytical expression is expected to work well on metals with free-electron-like valence bands, the performance of the analytical expression shown here is likely better than for most materials. There is a need for more experimentally acquired frequency-dependent photoemission data for a broad spectrum of materials beyond elemental metals to further validate this approach.

In conclusion, we have developed a generalizable DFTbased photoemission model, which has been demonstrated to quantitatively agree with experimental photoemission data for a diverse range of photocathode candidates. Coupled with the relatively low cost of DFT-based simulations, the general nature of our model may enable the rapid screening of thousands of photocathode candidates. Furthermore, we expect our model will pave the way for future studies on a wide variety of photonic materials, providing a fast and accurate method to explore novel photocathode and photoemissive technologies.

\section{ACKNOWLEDGMENTS}

This work was supported by the Department of Energy, Laboratory Directed Research and Development program at SLAC National Accelerator Laboratory, under Contract No. DE-AC02-76SF00515.
The Rh, W, and Mo experimental intrinsic emittance data were measured by G. Adhikari and W. A. Schroeder under National Science Foundation Award No. PHYS-1535279. The $\mathrm{Cu}$ experimental intrinsic emittance data were measured by G. Adhikari and S. Karkare under DOE Award No. DE-

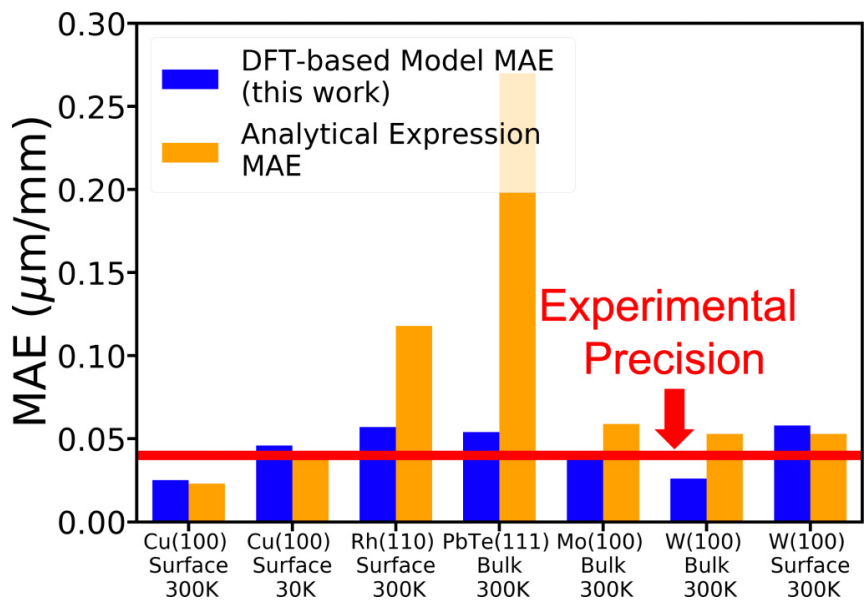

FIG. 9. Mean absolute error (MAE) of the intrinsic emittance calculated with our DFT-based method and the analytical expression [Eq. (5)] over the photon energy range of the experimental measurements, shown in Fig. 8. The MAE is calculated with respect to experimental measurements $[10,32,33,40]$. An approximate value of the experimental precision is also shown for Ref. [40]. 


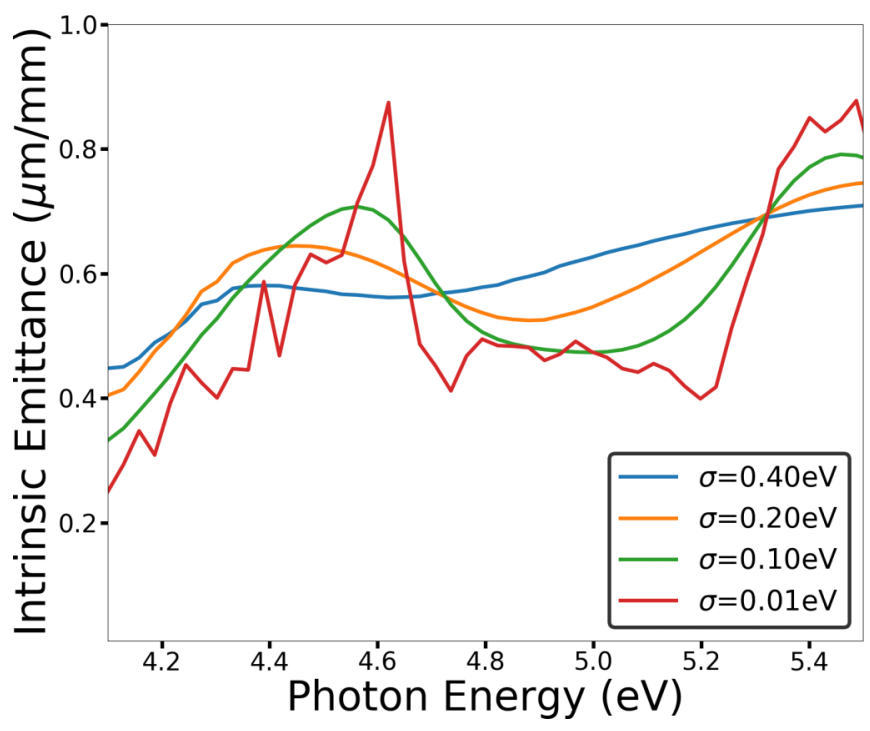

FIG. 10. Effect of $\sigma$ [Eq. (17)] on the calculated intrinsic emittance of bulk PbTe(111). We assume a lifetime broadening of $\sigma=$ $0.20 \mathrm{eV}$ for all intrinsic emittance calculations in this work.

SC0017621. P.S. gratefully acknowledges financial support from the Austrian Science Fund (FWF) under Contract No. J3980-N27.

\section{APPENDIX A: EFFECT OF $\sigma$ ON CALCULATED INTRINSIC EMITTANCE}

The Dirac delta function $\delta$ in Eq. (17) ensures the conservation of energy between the initial and final electronic states and the photon being absorbed. Physically, $\sigma$ in Eq. (17) represents the lifetime broadening of the excited states. Since $\sigma$ is difficult to calculate from DFT calculations, we solve Eq. (17) with an assumed value of $\sigma=0.20 \mathrm{eV}$. Figure 10 illustrates the calculated emittance of PbTe if we were to assume different values of $\sigma$. Larger values of $\sigma$ allow for more pairs of initial-final electronic states to have a larger contribution to the emittance, which smooths the data. However, even in the case of $\sigma=0.01 \mathrm{eV}$, the results are qualitatively similar to the case of $\sigma=0.20 \mathrm{eV}$.

\section{APPENDIX B: ALLOWABLE VALUES OF RECIPROCAL LATTICE VECTOR, $\vec{G}$}

To determine the range of excess energies that Eq. (30) can be used to describe the electron transmission probability, we calculate the maximum excess energy that prevents $G_{x, y} \neq 0$ from contributing to the photoemission [Eq. (31)] for Rh(110) (Fig. 11). $\mathrm{Rh}(110)$ is the material with the largest lattice constants included in this work, which gives a lower bound on the maximum excess energy that satisfies Eq. (31) for all studied materials. For $\mathrm{Rh}(110)$, the lowest excess energy that satisfies Eq. (31) with $G_{x, y} \neq 0$ is $2.54 \mathrm{eV}$, which is above the experimentally relevant excess energies. However, emission associated with $G_{x, y} \neq 0$ may occur in more complex crystals with larger lattice constants or surface reconstructions, leading to an increased emittance. (a)

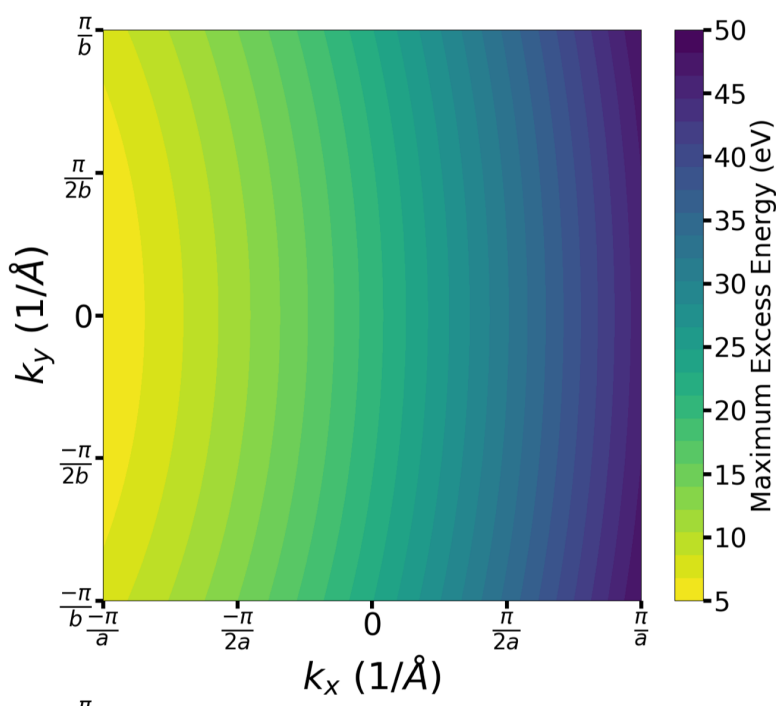

(b)

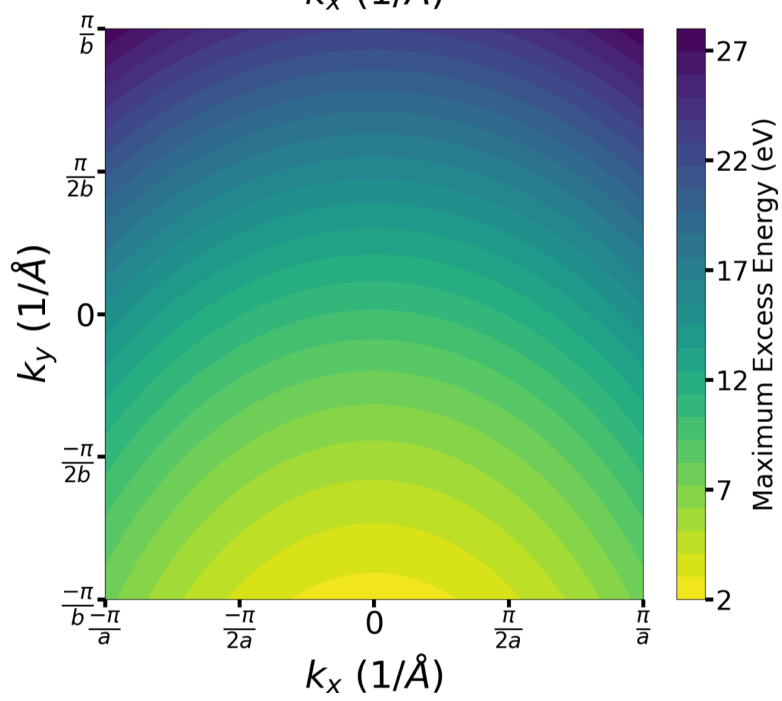

FIG. 11. Maximum excess energy $\left(E-V_{0}\right)$ that prevents $G_{x, y} \neq$ 0 terms of Eq. (29) from contributing to the photoemission of $\operatorname{Rh}(110)$ for (a) $G_{x}=\frac{2 \pi}{a}, G_{y}=0$ and (b) $G_{x}=0, G_{y}=\frac{2 \pi}{b}$.

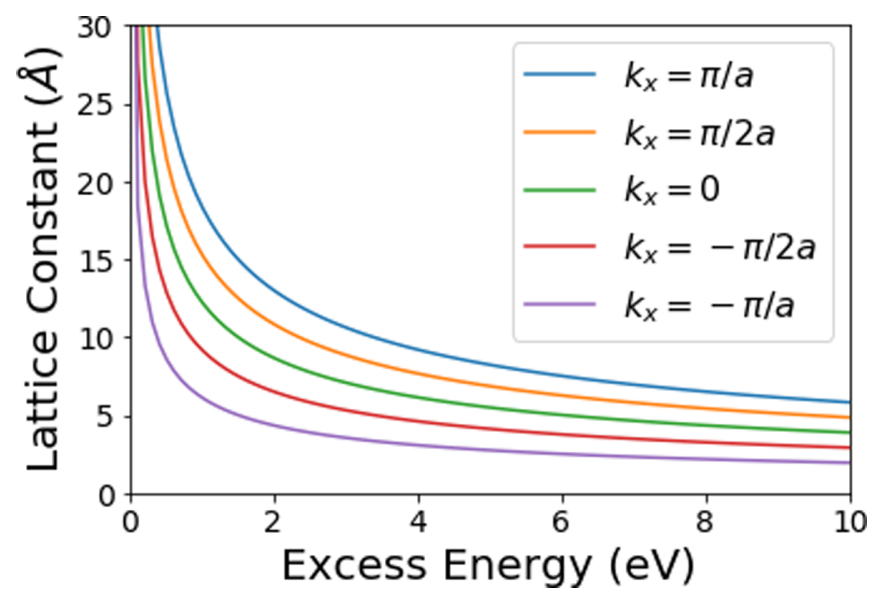

FIG. 12. Minimum lattice constant (a) of general photocathode materials that allow for $G_{x} \neq 0$ to contribute to the photoemission, with $G_{y}=0, k_{y}=0$ [Eq. (31)]. 
In general, we expect the $G_{x, y} \neq 0$ reciprocal-lattice vectors to contribute to the distribution of photoemitted electrons at lower excess energies for materials with larger lattice constants. To quantify this effect, we calculate the minimum lattice constant that we can ignore the contribution to the photoemission from $G_{x, y} \neq 0$, given by Eq. (31) (Fig. 12). For a typical range of experimental excess energies of up to $2 \mathrm{eV}$, $G_{x, y} \neq 0$ reciprocal-lattice vectors will not contribute to the photoemission for any material with a lattice constant smaller than $4.33 \AA$.
[1] E. Agrell, M. Karlsson, A. R. Chraplyvy, D. J. Richardson, P. M. Krummrich, P. Winzer, K. Roberts, J. K. Fischer, S. J. Savory, B. J. Eggleton, M. Secondini, F. R. Kschischang, A. Lord, J. Prat, I. Tomkos, J. E. Bowers, S. Srinivasan, M. BrandtPearce, and N. Gisin, Roadmap of optical communications, J. Opt. 18, 063002 (2016).

[2] F. P. García de Arquer, A. Armin, P. Meredith, and E. H. Sargent, Solution-processed semiconductors for nextgeneration photodetectors, Nat. Rev. Mater. 2, 16100 (2017).

[3] F. H. L. Koppens, T. Mueller, P. Avouris, A. C. Ferrari, M. S. Vitiello, and M. Polini, Photodetectors Based on Graphene, Other Two-Dimensional Materials and Hybrid Systems, Nat. Nanotechnol. 9, 780 (2014).

[4] M. W. Mara, R. G. Hadt, M. E. Reinhard, T. Kroll, H. Lim, R. W. Hartsock, R. Alonso-Mori, M. Chollet, J. M. Glownia, S. Nelson, D. Sokaras, K. Kunnus, K. O. Hodgson, B. Hedman, U. Bergmann, K. J. Gaffney, and E. I. Solomon, Metalloprotein entatic control of ligand-metal bonds quantified by ultrafast $\mathrm{x}$ ray spectroscopy, Science 356, 1276 (2017).

[5] E. M. Mannebach, C. Nyby, F. Ernst, Y. Zhou, J. Tolsma, Y. Li, M.-J. Sher, I.-C. Tung, H. Zhou, Q. Zhang, K. L. Seyler, G. Clark, Y. Lin, D. Zhu, J. M. Glownia, M. E. Kozina, S. Song, S. Nelson, A. Mehta, Y. Yu et al., Dynamic Optical Tuning of Interlayer Interactions in the Transition Metal Dichalcogenides, Nano Lett. 17, 7761 (2017).

[6] D. Kraus, J. Vorberger, A. Pak, N. J. Hartley, L. B. Fletcher, S. Frydrych, E. Galtier, E. J. Gamboa, D. O. Gericke, S. H. Glenzer, E. Granados, M. J. MacDonald, A. J. MacKinnon, E. E. McBride, I. Nam, P. Neumayer, M. Roth, A. M. Saunders, A. K. Schuster, P. Sun, T. van Driel, T. Döppner, and R. W. Falcone, Formation of diamonds in laser-compressed hydrocarbons at planetary interior conditions, Nat. Astron. 1, 606 (2017).

[7] R. Schoenlein, T. Elsaesser, K. Holldack, Z. Huang, H. Kapteyn, M. Murnane, and M. Woerner, Recent advances in ultrafast X-ray sources, Philos. Trans. R. Soc. Math. Phys. Eng. Sci. 377, 20180384 (2019).

[8] D. H. Dowell, I. Bazarov, B. Dunham, K. Harkay, C. Hernandez-Garcia, R. Legg, H. Padmore, T. Rao, J. Smedley, and W. Wan, Cathode R\&D for future light sources, Nucl. Instrum. Methods Phys. Res. Sect. Accel. Spectrometers Detect. Assoc. Equip. 622, 685 (2010).

[9] M. Xie, Design optimization for an x-ray free electron laser driven by SLAC linac, in Proceedings of Particle Accelerator Conference, Dallas, Texas (1995), pp. 183-185.

[10] J. K. Nangoi, T. Arias, S. Karkare, H. Padmore, and W. A. Schroeder, The role of electron-phonon scattering in transverse momentum conservation in $\mathrm{PbTe}(111)$ photocathodes, in IPAC 2018, Vancouver, Canada, (JACOW, Geneva, Switzerland, 2018 ), pp. 1414-1416.

[11] L. Cultrera, I. Bazarov, A. Bartnik, B. Dunham, S. Karkare, R. Merluzzi, and M. Nichols, Thermal emittance and response time of a cesium antimonide photocathode, Appl. Phys. Lett. 99, 152110 (2011).

[12] W. E. Spicer, Photoemissive, Photoconductive, and Optical Absorption Studies of Alkali-Antimony Compounds, Phys. Rev. 112, 114 (1958).

[13] D. H. Dowell and J. F. Schmerge, Quantum efficiency and thermal emittance of metal photocathodes, Phys. Rev. Spec. Top. - Accel. Beams 12, 074201 (2009).

[14] T. Vecchione and D. Dowell, Quantum efficiency and transverse momentum from metals, Proceedings of FEL2013, (New York, 2013)

[15] I. Bazarov, L. Cultrera, A. Bartnik, B. Dunham, S. Karkare, Y. Li, X. Liu, J. Maxson, and W. Roussel, Thermal emittance measurements of a cesium potassium antimonide photocathode, Appl. Phys. Lett. 98, 224101 (2011).

[16] T. Vecchione, Simulating Single Crystal Copper Photocathode Emittance, in Proceedings of FEL '15, Daejeon, Republic of Korea, (JACOW, Geneva, Switzerland, 2015), pp. 587-591.

[17] C. P. Hauri, R. Ganter, F. Le Pimpec, A. Trisorio, C. Ruchert, and H. H. Braun, Intrinsic Emittance Reduction of an Electron Beam from Metal Photocathodes, Phys. Rev. Lett. 104, 234802 (2010).

[18] T. Li and W. A. Schroeder, arXiv:1704.00194.

[19] P. Hohenberg and W. Kohn, Inhomogeneous Electron Gas, Phys. Rev. 136, B864 (1964).

[20] W. Kohn and L. J. Sham, Self-Consistent Equations Including Exchange and Correlation Effects, Phys. Rev. 140, A1133 (1965).

[21] M. Gajdoš, K. Hummer, G. Kresse, J. Furthmüller, and F. Bechstedt, Linear optical properties in the projector-augmented wave methodology, Phys. Rev. B 73, 045112 (2006).

[22] See Supplemental Material at http://link.aps.org/supplemental/ 10.1103/PhysRevB.101.235447 for video illustrating the photon energy dependence on the calculated intrinsic emittance of $\mathrm{PbTe}(111)$.

[23] G. Kresse and J. Furthmüller, Efficient iterative schemes for ab initio total-energy calculations using a plane-wave basis set, Phys. Rev. B 54, 11169 (1996).

[24] P. E. Blöchl, Projector augmented-wave method, Phys. Rev. B 50, 17953 (1994).

[25] A. Jain, S. P. Ong, G. Hautier, W. Chen, W. D. Richards, S. Dacek, S. Cholia, D. Gunter, D. Skinner, G. Ceder, and K. A. Persson, Commentary: The Materials Project: A materials genome approach to accelerating materials innovation, APL Mater. 1, 011002 (2013).

[26] J. P. Perdew, K. Burke, and M. Ernzerhof, Generalized Gradient Approximation Made Simple, Phys. Rev. Lett. 77, 3865 (1996).

[27] C. Cocchi, S. Mistry, M. Schmeißer, J. Kuhn, and T. Kamps, First-principles many-body study of the electronic and optical properties of CsK2 $\mathrm{Sb}$, a semiconducting material for ultra- 
bright electron sources, J. Phys.: Condens. Matter 31, 014002 (2018).

[28] C. Cocchi, S. Mistry, M. Schmeißer, R. Amador, J. Kuhn, and T. Kamps, Electronic structure and core electron fingerprints of caesium-based multi-alkali antimonides for ultra-bright electron sources, Sci. Rep. 9, 18276 (2019).

[29] R. N. Tauber, A. A. Machonis, and I. B. Cadoff, Thermal and Optical Energy Gaps in PbTe, J. Appl. Phys. 37, 4855 (1966).

[30] A. Goyal, P. Gorai, E. S. Toberer, and V. Stevanović, Firstprinciples calculation of intrinsic defect chemistry and selfdoping in PbTe, Npj Comput. Mater. 3, 42 (2017).

[31] R. Dalven, Energy-Gap Anomaly in the Semiconductor Sequence $\mathrm{PbS}, \mathrm{PbSe}$, and PbTe, Phys. Rev. B 3, 3359 (1971).

[32] S. Karkare, G. Adhikari, H. Padmore, T. Vecchione, and W. A. Schroeder, $<10 \mathrm{meV}$ MTE from $\mathrm{Cu}$, in Photocathode Physics for Photoinjectors (P3) 2018 Workshop, Santa Fe, New Mexico.

[33] W. A. Schroeder and G. Adhikari, Band Structure Interpretation of Mo(100) and W(100) Spectral Emission Properties, in Photocathode Physics for Photoinjectors (P3) 2018 Workshop, Santa Fe, New Mexico.
[34] D. E. Eastman, Photoelectric Work Functions of Transition, Rare-Earth, and Noble Metals, Phys. Rev. B 2, 1 (1970).

[35] M. G. Helander, M. T. Greiner, Z. B. Wang, and Z. H. Lu, Pitfalls in measuring work function using photoelectron spectroscopy, Appl. Surf. Sci. 256, 2602 (2010).

[36] S. De Waele, K. Lejaeghere, M. Sluydts, and S. Cottenier, Error estimates for density-functional theory predictions of surface energy and work function, Phys. Rev. B 94, 235418 (2016).

[37] K. Choudhary, Q. Zhang, A. C. E. Reid, S. Chowdhury, N. Van Nguyen, Z. Trautt, M. W. Newrock, F. Y. Congo, and F. Tavazza, Computational Screening of High-Performance Optoelectronic Materials Using OptB88vdW and TB-mBJ Formalisms, Sci. Data 5, 180082 (2018).

[38] W. E. Spicer and A. Herrera-Gomez, Modern theory and applications of photocathodes, Proc. SPIE 2022, 18 (1993).

[39] B. Camino, T. C. Q. Noakes, M. Surman, E. A. Seddon, and N. M. Harrison, Photoemission simulation for photocathode design: theory and application to copper and silver surfaces, Comput. Mater. Sci. 122, 331 (2016).

[40] G. Adhikari, P. Riley, and W. A. Schroeder, Spectral characterization of a $\mathrm{Rh}(110)$ photocathode: Band structure interpretation, AIP Adv. 9, 065305 (2019). 